\title{
Diagrammar of physical and fake particles and spectral optical theorem
}

\author{
Damiano Anselmi \\ Dipartimento di Fisica "Enrico Fermi", Università di Pisa, \\ Largo B. Pontecorvo 3, 56127 Pisa, Italy \\ INFN, Sezione di Pisa, \\ Largo B. Pontecorvo 3, 56127 Pisa, Italy \\ E-mail: damiano.anselmi@unipi.it
}

ABSTRACT: We prove spectral optical identities in quantum field theories of physical particles (defined by the Feynman $i \epsilon$ prescription) and purely virtual particles (defined by the fakeon prescription). The identities are derived by means of purely algebraic operations and hold for every (multi)threshold separately and for arbitrary frequencies. Their major significance is that they offer a deeper understanding on the problem of unitarity in quantum field theory. In particular, they apply to "skeleton" diagrams, before integrating on the space components of the loop momenta and the phase spaces. In turn, the skeleton diagrams obey a spectral optical theorem, which gives the usual optical theorem for amplitudes, once the integrals on the space components of the loop momenta and the phase spaces are restored. The fakeon prescription/projection is implemented by dropping the thresholds that involve fakeon frequencies. We give examples at one loop (bubble, triangle, box, pentagon and hexagon), two loops (triangle with "diagonal", box with diagonal) and arbitrarily many loops. We also derive formulas for the loop integrals with fakeons and relate them to the known formulas for the loop integrals with physical particles.

KeYwords: Beyond Standard Model, Models of Quantum Gravity

ArXiv EPrint: 2109.06889 


\section{Contents}

1 Introduction $\quad 2$

2 Definitions and strategy 3

2.1 Skeletons 5

2.2 Diagrammatic rules 8

2.3 Threshold decomposition 9

2.4 Identities for principal values 9

$\begin{array}{lll}2.5 & \text { Strategy } & 11\end{array}$

$\begin{array}{lll}2.6 & \text { Proper diagrammatic form and proper decomposition } & 12\end{array}$

$\begin{array}{lll}3 & \text { Bubbles } & 13\end{array}$

4 Triangle $\quad 16$

$\begin{array}{lll}4.1 & \text { Fakeons } & 18\end{array}$

$\begin{array}{llr}5 & \text { Box } & 19\end{array}$

5.1 Formulas 20

$\begin{array}{lll}5.2 & \text { Fakeons } & 21\end{array}$

$6 \quad$ Simple multiloop diagrams $\quad 22$

$\begin{array}{lll}7 & \text { Box with diagonal } & 23\end{array}$

$\begin{array}{lll}7.1 & \text { Fakeons } & 25\end{array}$

8 Further insight into the algebraic structure of the spectral optical iden$\begin{array}{ll}\text { tities } & 26\end{array}$

9 Generalization to propagators with arbitrary real residues $\quad 28$

10 Diagrams with nontrivial numerators and degenerate diagrams $\quad 29$

11 Thick fakeons $\quad 31$

12 Massless fields and infrared divergences $\quad 34$

$\begin{array}{ll}13 \text { Conclusions } & 36\end{array}$ 


\section{Introduction}

Unitarity is a key requirement to claim that a quantum field theory has chances to be fundamental, together with locality and renormalizability. Generically, locality and renormalizability can be phrased in simple terms: the Lagrangian should be polynomial in the fields and their derivatives and obey the power counting criterion. Moreover, the theorem of locality of counterterms shows that there is a nontrivial connection between renormalizability and locality. Unitarity, on the other hand, still lacks an understanding in equally simple terms. In this paper we overcome this drawback and extend the classical results $[1-3,5,6]$ in several directions.

We show that unitarity in quantum field theory can be reduced to a set of algebraic identities, which do not require to integrate on the space components of the loop momenta, or the phase spaces in cut diagrams, and hold for each physical threshold separately. The key ingredient is a proper threshold decomposition, because different thresholds do not talk to one another.

The gain in insight is important. Among the other things, we can identify the purely virtual (off-shell) content of amplitudes and diagrams. We can switch from the Feynman prescription to the fakeon prescription $[7,8]$ by simply dropping all the thresholds that involve one or more frequencies associated with the legs that we want to quantize as fakeons (that is to say, purely virtual particles). We can easily generalize the identities to propagators with arbitrary residues and arbitrary frequencies, as well as vertices with arbitrary derivative structures (as long as the classical Lagrangian stays Hermitian). We can show that the spectral identities hold for thick fakeons as well (fakeons with finite "widths" at the tree level ${ }^{1}$ ), which are typically originated by higher-derivative Lagrangians and can be used to reformulate the Lee-Wick models [10-15] as models of particles and fakeons $[16,17]$. We can even introduce certain details of the experimental apparatus without violating the spectral optical identities. For example, we can prove unitarity in the presence of fakeons when the energy resolution of detectors is taken into account, as a cutoff for the infrared divergences of massless fields [18-21]. It is also possible to include the energy resolution of fakeons in a unitary way.

Purely virtual particles [22], or "fakeons", can be used to formulate a consistent theory of quantum gravity [7], which is experimentally testable thanks to its predictions in inflationary cosmology [23]. More generally, they can be used to solve the problem of ghosts in higher-derivatives theories. However, they can also be employed in collider physics [24] to evade common constraints and offer new ways to solve discrepancies with data, as in the problem of the muon anomalous magnetic moment [25].

Everything we do in this paper involves nothing more than purely algebraic operations. Despite some unavoidably lengthy expressions, the identities we write can be checked straightforwardly. First, we integrate on the loop energies. This is the only integral we need to do. We can view it as an algebraic operation as well, thanks to the residue theorem. Then, we ignore the integrals on the space components of the loop momenta and the phase spaces, i.e., work on the "skeleton" of the diagram. Third, we eliminate the pseudothresh-

\footnotetext{
${ }^{1}$ For a review, see $[9]$.
} 
olds, which are unphysical, because they involve differences of frequencies. Once we remain with the physical thresholds, we proceed with the threshold decomposition, which provides a separate optical identity for each threshold. Summing the identities associated with a loop diagram, we derive the spectral optical theorem obeyed by its skeleton. Integrating the spectral optical theorem on the space components of the loop momenta and the phase spaces, we obtain the usual optical theorem for amplitudes, which proves unitarity.

Besides proving the identities and the spectral optical theorem for arbitrary diagrams, we give a large number of examples that show how the threshold decomposition works. Apart from the bubble and the triangle, which are relatively straightforward, we study the box, the pentagon and the hexagon, at one loop. At two loops we study the triangle with "diagonal" and the box with diagonal. These examples cover the needs of most calculations in high-energy physics phenomenology. Whole classes of diagrams with arbitrarily many loops are included straightforwardly. The formulas we obtain for the loop integrals with fakeons and physical particles can be related to the formulas of ordinary loop integrals (defined by the Feynman prescription everywhere) and implemented in softwares like FeynCalc, FormCalc, LoopTools and Package-X [26-32].

The paper is organized as follows. In section 2 we collect the basic definitions and the strategy of the calculations. In sections 3,4 and 5 we study the bubble diagram (and its multibubble versions), the triangle diagram and the box diagram, respectively. In section 6 we extend the results to classes of multiloop diagrams. In section 7 we study the box diagram with diagonal. In section 8 we provide further insight into the algebraic structure of the spectral optical identities and derive the formulas of the pentagon and the hexagon. In section 9 we generalize the results to propagators with arbitrary residues. In section 10 we extend the proof to diagrams with derivative vertices (integrands with nontrivial numerators) and degenerate diagrams (diagrams with powers of identical propagators or subdiagrams). In section 11 we generalize the proofs to propagators with thick fakeons. In section 12 we show how to treat massless fields and their infrared divergences. Section 13 contains the conclusions.

\section{Definitions and strategy}

In this section we collect the basic definitions we need and the strategy of the calculations. Since the matter is technically involved, not all the subtleties we anticipate here can be appreciated right away. Nevertheless, it is convenient to collect them in a dedicated place to allow a quick back and forth, for a better understanding of the next sections.

Unitarity is the statement that the scattering matrix ( $S$ matrix) is unitary. Writing $S=1+i T$, where $T$ is the transition amplitude (and $i T$ is the sum of loop diagrams), we can write the unitarity equation $S^{\dagger} S=1$ in the equivalent form

$$
2 \operatorname{Im} T=T^{\dagger} T
$$

which is known as optical theorem. Proving unitarity, or the optical theorem, is crucial to establish that a quantum field theory is consistent as a fundamental theory. 

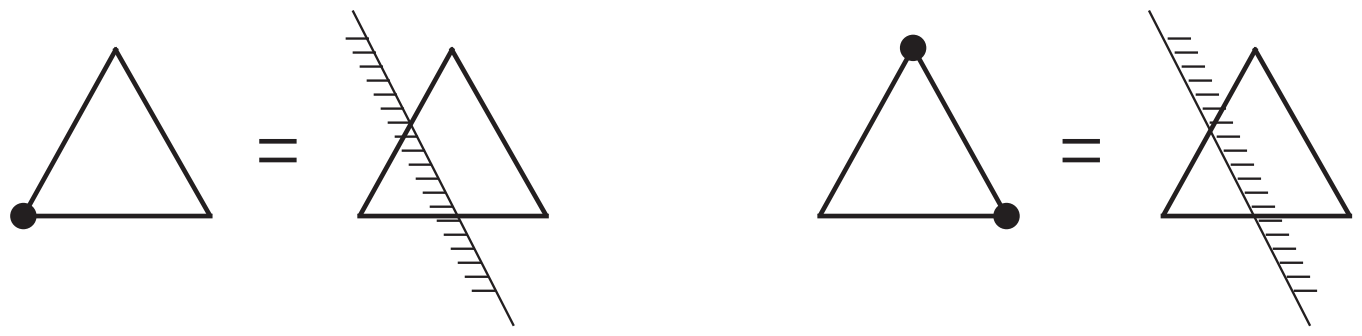

Figure 1. Marked diagrams and cut diagrams. The shadowed portion stands for $T^{\dagger}$, the unshadowed one for $T$.

More powerful statements can be proved, in general, such as diagrammatic versions of (2.1), which hold for each loop integral separately. They can be expressed as relations

$$
G+\bar{G}+\sum_{c} G_{c}=0
$$

among a given diagram $G$, its complex conjugate $\bar{G}$ and certain "cut diagrams" $G_{c}$. The cut diagrams are diagrams divided into two portions by a cut passing through a number of propagators (which become "cut propagators"). One portion stands of the $T$ of (2.1) and the other one (which is normally shadowed) stands for $T^{\dagger}$. Like $G$ and $\bar{G}$, the cut diagrams can be defined by means of diagrammatic rules, which, in addition to the ordinary propagators and vertices, involve cut propagators, complex conjugate propagators and complex conjugate vertices. The rules are given in subsection 2.2.

In ref. [33] it was shown that the diagrammatic version (2.2) of the optical theorem can be derived algebraically and expressed by means of algebraic equations, which do not require to integrate on the space components of the loop momenta or phases spaces. ${ }^{2}$ In this paper we exploit these properties to the fullest.

As in [33], it is convenient to denote the cut diagrams in "dual" form, by means of marked and unmarked vertices. In figure 1 we show the relation between marked diagrams and cut diagrams. In figure 2 we show the marked versions of the triangle diagram, up to permutations of the vertices.

This convention allows us to identify the diagrams by means of simple "words". Let A, B, C, etc., label the unmarked vertices and $\dot{\mathrm{A}}, \dot{\mathrm{B}}, \dot{\mathrm{C}}$, etc., the marked vertices. The propagators are segments. Segments like AB, BC, etc., are ordinary propagators, while segments like $\dot{A} \dot{B}, \dot{B} \dot{C}$, etc., are complex conjugate propagators. Segments like $\dot{A} B, B \dot{C}$, etc., are cut propagators with positive energies flowing from the vertices without the dot to the vertices with the dot.

The loops are enclosed between parentheses. A one-loop (cut or uncut) diagram is a "word" between parentheses, built with letters. For example: $(\mathrm{AB})$ is the uncut bubble diagram $\bigvee$, where A denotes the left vertex and B is the right vertex. Then, $(\dot{\mathrm{A}} \dot{\mathrm{B}})$ denotes

\footnotetext{
${ }^{2}$ Some integrals on the loop space momenta of $G$ and $\bar{G}$ appear as integrals on phase spaces in $G_{c}$. They are those interested by the cut propagators, which put the particles on shell. They stand for the contraction between $T^{\dagger}$ and $T$ of formula (2.1).
} 


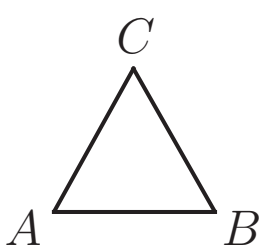

$(\mathrm{ABC})$

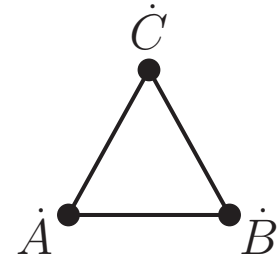

$(\dot{\mathrm{A}} \dot{\mathrm{B}} \dot{\mathrm{C}})$

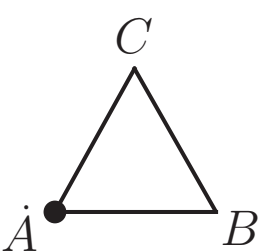

$(\dot{\mathrm{ABC}})$

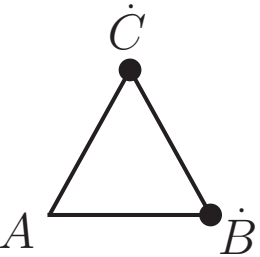

$(\mathrm{AB} \dot{\mathrm{C}})$

Figure 2. Triangle uncut and cut diagrams (in marked notation).

the complex conjugate diagram, while $(\dot{\mathrm{AB}})$ denotes the cut diagram with positive energies flowing from $\mathrm{B}$ to $\mathrm{A}$ and $(\mathrm{AB})$ is the cut diagram with positive energies flowing from $\mathrm{A}$ to $\mathrm{B}$.

Similarly, $(\mathrm{ABC})$ is the uncut triangle diagram, $(\dot{\mathrm{A}} \dot{\mathrm{B}} \dot{\mathrm{C}})$ is its complex conjugate, $(\dot{\mathrm{A} B C})$ is the cut diagram with positive energies flowing to $\mathrm{A}$, etc.

Diagrams with more loops are denoted by means of products of one-loop words in parentheses, repeating the vertices that belong to more loops. For example, the double bubble $\mathrm{OO} \alpha$ is $(\mathrm{AB})(\mathrm{BC})$, its conjugate is $(\dot{\mathrm{A}} \dot{\mathrm{B}})(\dot{\mathrm{B}} \dot{\mathrm{C}})$, its cut versions are $(\dot{\mathrm{AB}})(\mathrm{BC})$, $(\mathrm{AB})(\dot{\mathrm{B} C})$, etc.

The legs are labeled by numbers: the box diagram, which is concisely denoted by $(\mathrm{ABCD})$, can be denoted by (A1B2C3D4) in extended form, where 1 is the leg $\mathrm{AB}, 2$ is the leg BC, 3 is the leg CD and 4 is the leg DA. Similarly, (AB)(BC) may stand for (A1B2)(B3C4), etc. Different "words" may correspond to the same diagram, but this is not going to cause trouble.

The diagrammatic optical theorem (2.2) is the statement that, given a diagram $G$, the sum of all its marked versions (including $G$ itself) vanishes. For example, in the case of the triangle the sum of the diagrams shown in figure 2 plus the cyclic permutations of the last two is equal to zero. Here we generalize the theorem to spectral optical identities, which separately hold for each threshold. To achieve this result, we need to work out a neat threshold decomposition of the "skeleton" diagrams, defined below.

In the first part of our investigation, we concentrate on the loop integrals defined by the Feynman $i \epsilon$ prescription everywhere. As soon as their threshold decomposition is worked out, it is relatively straightforward to apply the fakeon prescription/projection to it.

We do not need to handle the ultraviolet divergences, but, for the sake of precision, we understand that the loop integrals are defined by means of the dimensional regularization [37-39] as follows: the integrals on the loop energies are defined by the residue theorem, while the integrals on the space components of the loop momenta are defined as the dimensionally regularized ones in $D-1$ continued dimensions.

\section{$2.1 \quad$ Skeletons}

The only integrals we compute in this paper are those on the loop energies. Their evaluation amounts to an algebraic operation, thanks to the residue theorem. If we drop the integrals over the space components of the loop momenta (and the phase spaces, in cut diagrams), as well as certain overall factors, we obtain the skeletons. 
If $G(p)$ denotes an ordinary loop integral (with all the propagators defined by the Feynman $i \epsilon$ prescription), the skeleton $G^{s}$ of $G$ is defined by the formula

$$
G(p)=\int \prod_{l=1}^{L} \frac{\mathrm{d}^{D-1} \mathbf{k}_{l}}{(2 \pi)^{D-1}}\left(\prod_{a=1}^{I} \frac{1}{2 \omega_{a}}\right) G^{s}(p, \mathbf{k}),
$$

where $p_{a}^{\mu}=\left(e_{a}, \mathbf{p}_{a}\right)$ are the external momenta (one for each internal leg), $k_{l}^{\mu}=\left(k_{l}^{0}, \mathbf{k}_{l}\right)$ are the loop momenta, $L$ is the number of loops, $I$ is the number of internal legs and $\omega_{a}$ is the frequency associated with the ath internal leg.

For example, the basic one-loop integrals

$$
G_{N}=\int \frac{\mathrm{d}^{D} k}{(2 \pi)^{D}} \prod_{a=1}^{N} \frac{1}{\left(k-p_{a}\right)^{2}-m_{a}^{2}+i \epsilon_{a}}
$$

define the one-loop skeletons

$$
G_{N}^{s}=\int \frac{\mathrm{d} k^{0}}{2 \pi} \prod_{a=1}^{N} \frac{2 \omega_{a}}{\left(k-p_{a}\right)^{2}-m_{a}^{2}+i \epsilon_{a}}=\int \frac{\mathrm{d} k^{0}}{2 \pi} \prod_{a=1}^{N} \frac{2 \omega_{a}}{\left(k^{0}-e_{a}\right)^{2}-\omega_{a}^{2}+i \epsilon_{a}}
$$

so that

$$
G_{N}=\int \frac{\mathrm{d}^{D-1} \mathbf{k}}{(2 \pi)^{D-1}}\left(\prod_{a=1}^{N} \frac{1}{2 \omega_{a}}\right) G_{N}^{s}
$$

where the frequencies are $\omega_{a}=\sqrt{\left(\mathbf{k}-\mathbf{p}_{a}\right)^{2}+m_{a}^{2}}$.

Note that $L$ momenta $p_{a}$ are redundant, since they can be eliminated by translating the loop momenta. The redundant notation allows us to treat all the internal legs equally, which is more convenient for the derivations of this paper.

Given complexified external momenta $p$, if the skeleton $G^{s}(p, \mathbf{k})$ is regular for every values of the space components $\mathbf{k}_{l}$ of the loop momenta within their integration domains, then $G(p)$ is analytic in $p$. The most important analytic region is the Euclidean one, where the energies are imaginary.

The $p$ domains where $G(p)$ is not analytic are found by studying the singularities of $G^{s}(p, \mathbf{k})$. Important domains of non analyticity are identified by inequalities of the form

$$
P_{E}^{2} \geqslant\left(\sum_{a \in J} m_{a}\right)^{2}
$$

where $P_{E}$ is a linear combination of external momenta and $J$ is a subset of internal legs. The right-hand side of this formula is an optical threshold, namely the minimum invariant mass of a physical process where the external particles involved in $P_{E}$ produce the internal particles of the subset $J$, turning them from virtual to real. ${ }^{3}$ These thresholds are called "optical", because they contribute to the optical theorem. Other physical thresholds exist, which do not participate in the optical theorem. They are called anomalous thresholds [34, 35].

\footnotetext{
${ }^{3} \mathrm{~A}$ fakeon is purely virtual, i.e., it can never become real. If a leg of the set $J$ is defined as a fakeon (see below for details), the threshold appearing on the right-hand side of (2.7) is not associated with a physical process. It is associated with the mathematical violation of analyticity and other physical properties, typical of fakeons, which we do not need to detail here. We may call it a "fake threshold" and say that it is associated with a fake physical process.
} 
After integrating on the loop energies, the skeletons are sums of terms that involve denominators of the forms

$$
\tilde{D}_{\text {phys }}=E+\sum_{a \in J} \tilde{\omega}_{a}, \quad \tilde{D}_{\text {pseudo }}=E+\sum_{a \in J} \tilde{\omega}_{a}-\sum_{b \in J^{\prime}} \tilde{\omega}_{b}
$$

where $\tilde{\omega}_{a}=\omega_{a}-i \epsilon_{a}, E$ is a linear combination of the external energies $e_{c}$ and $J, J^{\prime}$ are nonempty subsets of internal legs. Defining

$$
D_{\text {phys }}=\left.\tilde{D}_{\text {phys }}\right|_{\epsilon_{a} \rightarrow 0}, \quad D_{\text {pseudo }}=\left.\tilde{D}_{\text {pseudo }}\right|_{\epsilon_{a} \rightarrow 0},
$$

the zeros of $D_{\text {phys }}$ are associated with the physical thresholds, while the zeros of $D_{\text {pseudo }}$ are associated with the so-called pseudothresholds. The $i \epsilon$ prescription on the loop energies picks the right residues, which ultimately make the pseudothresholds cancel out. ${ }^{4}$ To have control on the pseudothresholds in the calculations, we must choose the infinitesimal widths $\epsilon_{a}$ such that all the denominators $\tilde{D}_{\text {pseudo }}$ have nonvanishing imaginary parts (otherwise they are not well prescribed).

The linear combinations of energies and frequencies generated by the diagrammatics have coefficients 0 and \pm 1 . This is guaranteed by the fact that an energy cannot flow into the same line twice (assuming a "minimal" parametrization of the momenta, such that every loop momentum $k_{l}$ appears in some leg $a$ with momentum $p_{a}-k_{l}$ ). All the manipulations we make in this paper involve linear combinations of the type just mentioned.

Moreover, the operations we make on skeletons are manifestly Lorentz invariant once we resume the integrals over the space components of the loop momenta and the phase spaces. The thresholds (2.7) emerge after such integrals. Since we work on skeletons here, from now we understand that the words "singularity", "threshold" and "pseudothreshold" may also refer to the skeletons.

Not all types of $G^{s}(p, \mathbf{k})$ singularities can be generated, but only "diagrammatic" ones. As said, the potential violations of analyticity occur when one or more skeleton denominators vanish. Since the $G^{s}(p, \mathbf{k})$ denominators are originated by the diagrammatics, it must be possible to express the singularities diagrammatically. This means that, if we restore the integrals on the loop energies, the loop energies circulate in the diagram in the way prescribed by the diagrammatics. In some manipulations, we may create contributions with non diagrammatic singularities, which cancel out in the total. We give an example of a non diagrammatic singularity in formula (2.24). We illustrate the cancellation of the non diagrammatic singularities when we investigate the box (section 5) and the box with diagonal (section 7 ).

The spectral optical theorem we are going to prove reads

$$
G^{s}+\bar{G}^{s}+\sum_{c} G_{c}^{s}=0
$$

where $G^{s}, \bar{G}^{s}$ and $G_{c}^{s}$ are the skeletons associated with $G, \bar{G}$ and $G_{c}$, respectively. The spectral optical identities are the threshold contributions to $(2.10)$, which vanish separately.

\footnotetext{
${ }^{4}$ The physical reason why the denominators $D_{\text {pseudo }}$ must disappear is that the differences of frequencies contained in them would lead to processes plagued by instabilities.
} 


\subsection{Diagrammatic rules}

It is convenient to think of each propagator as the sum of two separate contributions, one for each pole:

$$
\frac{i}{p^{2}-m^{2}+i \epsilon} \rightarrow \frac{i}{2 \omega}\left(\frac{1}{e-\omega+i \epsilon}-\frac{1}{e+\omega-i \epsilon}\right)
$$

where $p^{\mu}=(e, \mathbf{p})$ and $\omega=\sqrt{\mathbf{p}^{2}+m^{2}}$. The arrow means that we are ignoring contributions that disappear when $\epsilon$ tends to zero. If we write all the propagators as shown on the righthand side of (2.11), we can expand the integrand of a diagram as a sum of terms where each internal leg is associated with a unique pole. This is very convenient for the analysis of this paper.

Since we work with skeleton diagrams, without integrating over the space components of the loop momenta or phase spaces, we are free to multiply the propagators by $\omega$-dependent overall factors. We choose such factors equal to $2 \omega$, to match the definition of skeletons given above. The propagators and cut propagators of physical particles are then

$$
\begin{aligned}
\frac{p \rightarrow}{p \rightarrow} & =\frac{i}{e-\omega+i \epsilon}-\frac{i}{e+\omega-i \epsilon}, \\
\bullet & =\frac{i}{e+\omega+i \epsilon}-\frac{i}{e-\omega-i \epsilon}, \\
\square \rightarrow \quad \leftarrow p & =(2 \pi) \delta(e+\omega), \quad \leftarrow \quad \leftarrow 2 \pi) \delta(e-\omega) .
\end{aligned}
$$

For definiteness, we assume that each unmarked vertex is equal to $-i$. Then, each marked vertex is equal to $i$. In section 10 we show how to extend the results to derivative vertices.

We consider connected, non necessarily one-particle irreducible (1PI) diagrams $G$. If $G$ is disconnected by cutting an internal line $\hat{I}$, we say that $\hat{I}$ is a "link". Inside cut diagrams $G_{c}$ we apply this definition to both sides of the cut separately. Thus, if one side of the cut is disconnected when a certain leg is broken, we call that leg a link.

Now we give the basic diagrammatic rules for the internal legs that we want to define as fakeons (denoted by "f"). The cut propagators must vanish:

$$
\text { - } \frac{p \rightarrow}{\mathrm{f}}=0, \quad \frac{\leftarrow p}{\mathrm{f}}=0
$$

The propagator of a link fakeon leg is just the Cauchy principal value $\mathcal{P}$ (which is the classical limit of fakeon prescription [9]):

$$
\begin{aligned}
(\text { link }): & \frac{p \rightarrow}{\mathrm{f}}=\mathcal{P}\left(\frac{i}{e-\omega}-\frac{i}{e+\omega}\right), \\
(\text { link }): & \stackrel{p \rightarrow}{\mathrm{f}} \cdot=\mathcal{P}\left(\frac{i}{e+\omega}-\frac{i}{e-\omega}\right) .
\end{aligned}
$$

As for the other fakeon legs, the fakeon prescription will be implemented after deriving the spectral optical identities of $G^{s}$, by dropping the thresholds that involve frequencies $\omega$ associated with fakeon legs. 
Note that it is incorrect to use (2.14) inside 1PI diagrams [22]. What we have to do, instead, is apply the strategy explained in subsection 2.5.

\subsection{Threshold decomposition}

The crucial operation is the threshold decomposition of a diagram, which we briefly describe here, leaving the details to next subsections.

After integrating on the loop energies by means of the residue theorem, we obtain the skeletons. Then, we use certain algebraic manipulations to make all the pseudothresholds $D_{\text {pseudo }}=0$ disappear and only the physical thresholds $D_{\text {phys }}=0$ survive. At that point we apply the decomposition

$$
\frac{i}{x+i \epsilon}=\mathcal{P} \frac{i}{x}+\pi \delta(x)
$$

and reorganize the results diagrammatically (see below).

Once these operations are completed, we obtain terms proportional $\delta$ functions and terms proportional to products of $\delta$ functions. The terms proportional to one $\delta$ function are called single thresholds, while those proportional to a product of more $\delta$ functions are called multithresholds. When we want to be more precise, we call the latter double thresholds, triple thresholds, etc., or $\ell$ thresholds, where $\ell$ is the "level" of the threshold. The support of the $\delta$ functions is the support of the threshold. Sometimes the word "threshold" is used indistinctly for all of them. The "zero threshold" collects the contributions that contain only principal values, which identify the purely virtual content of the diagram. The zeros of the principal values are called "singularities".

In the end, the threshold decomposition is the decomposition that properly organizes and separates the independent thresholds and singularities. We stress again that they must be physical (no $D_{\text {pseudo }}$ can survive) and diagrammatic (because generated by diagrams).

The results of the decomposition are often collected in a table (see table 2 for the triangle) with the following structure. The columns collect the contributions of the (cut or uncut) skeleton diagrams shown at the top of them. The rows collect the contributions of the different (multi)thresholds. The rows are ordered according to the threshold level, from the zeroth level at the top to the highest level at the bottom. The smaller tables are shown as equations, while the larger tables are displayed separately, on top of pages.

The spectral optical identities are the sums of the entries of the rows of a table. They vanish separately, because each row corresponds to a different threshold and different thresholds do not interfere with one another. The spectral optical theorem is obtained by collecting all the entries of the table in the way explained below. The usual optical theorem is obtained by integrating the spectral optical theorem (divided by $2 \omega$ for every internal leg) over the phase spaces and the space components of the loop momenta.

\subsection{Identities for principal values}

We show a simple application of the threshold decomposition just described, which gives identities that are useful for the calculations of the paper. Consider the expression

$$
S\left(x_{1}, \ldots, x_{n}\right)=\prod_{i=1}^{n} \frac{1}{x_{i}}-\sum_{i=1}^{n}\left(\prod_{j=1, j \neq i}^{n} \frac{1}{x_{j}}\right)\left(\sum_{l=1}^{n} x_{l}\right)^{-1} \text {. }
$$


If we sum the right-hand side with the least common denominator, we find zero. This operation is legitimate only if the variables $x_{i}$ and their sum do not vanish. It is sufficient to assume that they have nonvanishing imaginary parts $\sigma_{i}$ (typically brought by the infinitesimal widths $\epsilon_{a}$ ) such that $\sum_{i=1}^{n} \sigma_{i} \neq 0$.

It is not legitimate to infer $S\left(x_{1}, \ldots, x_{n}\right)=0$ if the variables $x_{i}$ have real values, in general. In particular, the principal value $\mathcal{P} S\left(x_{1}, \ldots, x_{n}\right)$ may not vanish. To work out its expression, we start from the identity

$$
0=S\left(x_{1}+i \epsilon_{1}, \ldots, x_{n}+i \epsilon_{n}\right)
$$

where the $x_{j}$ are now real and $\epsilon_{j}>0$ for every $j$. Expanding each term on the righthand side of (2.16) by means of (2.15), we can derive its threshold decomposition. The lowest level of the decomposition is the principal value $\mathcal{P} S\left(x_{1}, \ldots, x_{n}\right)$ and the highest level involves only $\delta$ functions. It is easy to show that (2.17) gives an identity

$$
\mathcal{P} S\left(x_{1}, \ldots, x_{n}\right)=c_{n} \pi^{n} \prod_{i=1}^{n} \delta\left(x_{i}\right),
$$

where $c_{n}$ is a real constant. We can prove this statement iteratively in $n$. For $n=1$ the identities (2.17) and (2.18) are trivial. Assuming that the statement holds for $n=\hat{n}$, let us consider the case $n=\hat{n}+1$. It is easy to show that all the levels of the threshold decomposition but the lowest and highest ones can be converted into products of $\delta$ functions by using identities $(2.18)$ with $n \leqslant \hat{n}$. Thus, the lowest and highest levels must be related by an identity (2.18) with $n=\hat{n}+1$.

Note that, by reality, $\mathcal{P} S\left(x_{1}, \ldots, x_{n}\right)=0$ for every odd $n$.

Among the identities (2.18), we mention the cases $n=2,3,4$, which will be useful in the next sections. We find

$$
\begin{aligned}
\mathcal{P}\left(\frac{1}{x y}-\frac{1}{x(x+y)}-\frac{1}{y(x+y)}\right) & =-\pi^{2} \delta(x) \delta(y) \\
\mathcal{P}\left[\frac{1}{x y z}-\frac{1}{x+y+z}\left(\frac{1}{x y}+\frac{1}{x z}+\frac{1}{y z}\right)\right] & =0 \\
\mathcal{P}\left[\frac{1}{x y z w}-\frac{1}{x+y+z+w}\left(\frac{1}{x y z}+\frac{1}{x y w}+\frac{1}{x z w}+\frac{1}{y z w}\right)\right] & =\pi^{4} \delta(x) \delta(y) \delta(z) \delta(w) .
\end{aligned}
$$

A good exercise is to check the steps of the proof outlined above in these examples. It is also straightforward to verify the identities (2.19) numerically, with the help of arbitrary test functions $\varphi\left(x_{1}, \ldots, x_{n}\right)$.

The reason why we cannot use common denominators in the expressions between brackets and conclude that the total is zero is that common denominators worsen the singularities. Even when the numerators are formally zero, the total can be nonvanishing.

The identities just found are useful to work out the correct threshold decomposition. In particular, they allow us to remove all the spurious thresholds and singularities, which are the non diagrammatic ones and those due to the denominators $D_{\text {pseudo }}$. The identities must be applied within the same 1PI diagram. Connected, non 1PI diagrams are treated by viewing them as products of 1 PI subdiagrams (see below). 


\subsection{Strategy}

For future use, it is convenient to define

$$
\begin{aligned}
F^{a b} & =\frac{1}{e_{a}-e_{b}-\tilde{\omega}_{a}-\tilde{\omega}_{b}}, & \mathcal{P}^{a b} & =\mathcal{P} \frac{1}{e_{a}-e_{b}-\omega_{a}} \\
\mathcal{Q}^{a b} & =\mathcal{P}^{a b}-\mathcal{P} \frac{1}{e_{a}-e_{b}-\omega_{a}+\omega_{b}}, & & \hat{\mathcal{Q}}^{a b}=\left.\mathcal{Q}^{a b}\right|_{\omega_{a} \rightarrow-\omega_{a}}, \\
\Delta^{a b} & =\pi \delta\left(e_{a}-e_{b}-\omega_{a}-\omega_{b}\right), & &
\end{aligned}
$$

where we recall that $\omega_{a}$ are frequencies and $\tilde{\omega}_{a}=\omega_{a}-i \epsilon_{a}$.

It is also useful to introduce some "smart" manipulations. A "smart" common denominator identity is an identity of the form

$$
\sum_{i} c_{i} \prod_{j=1}^{n} \frac{1}{\tilde{D}_{i j}}=\sum_{l} c_{l}^{\prime} \prod_{j=1}^{n} \frac{1}{\tilde{D}_{\text {phys } l j}},
$$

where $c_{i}$ and $c_{l}^{\prime}$ are numerical coefficients and each $\tilde{D}_{i j}$ can be a $\tilde{D}_{\text {phys }}$ or a $\tilde{D}_{\text {pseudo }}$. Observe that each term of (2.21) has the same number $n$ of denominators. Also recall that all the manipulations we make involve linear combinations of energies and frequencies with coefficients $0,+1$ or -1 . An example of (2.21) is the identity

$$
\frac{F^{12}-F^{13}}{e_{2}-e_{3}+\tilde{\omega}_{2}-\tilde{\omega}_{3}}=F^{12} F^{13}
$$

taken from (2.17) with $n=2$.

If the left-hand side of (2.21) is generated by a diagram, with all the legs prescribed à la Feynman, it is always possible to remove the denominators $\tilde{D}_{\text {pseudo }}$ and obtain a sum of terms like the right-hand side. This is a key property of the Feynman prescription on the energies, which guarantees (perturbative) stability.

The strategy of the calculations is as follows. We assume, for simplicity, that $G$ is oneparticle irreducible, the extension to connected, non 1PI diagrams being straightforward.

1) Start from the diagram $G$, prescribed à la Feynman.

2) Integrate on the loop energies by means of the residue theorem.

3) Use smart common-denominator identities (2.21) to remove all the denominators $\tilde{D}_{\text {pseudo }}$ and leave only denominators $\tilde{D}_{\text {phys }}$.

4) Perform the threshold decomposition as follows:

4.a) separate each fraction $1 / \tilde{D}_{\text {phys }}$ into its purely virtual part and its on-shell part by means of (2.15);

4.b) use identities for principal values such as (2.19) to arrange the decomposition in a proper diagrammatic form (see below for the meaning of this).

5) Perform the fakeon projection, by eliminating all the thresholds that involve fakeon frequencies (which will be called "fakeon thresholds"). 
Steps 1) to 4) lead to the spectral optical identities of the diagrams where each leg is prescribed à la Feynman. If some legs are prescribed as fakeons, the identities reduce to a proper subset, as per step 5).

The purely virtual content of a diagram is its level 0 . It is associated with the diagram where all the internal legs are fakeons. Away from the thresholds, it coincides with the Euclidean version of the skeleton. It admits no nontrivial cuts.

Due to the diagrammatic structure mentioned in point 4.b), all the threshold contributions (level $\geqslant 1$ ) are manifestly Lorentz invariant, once we integrate on the space components of the loop momenta and the phase spaces. By subtraction, the purely virtual part is also Lorentz invariant, although not manifestly.

The threshold decomposition also applies to cut diagrams. There, step 2) is simplified by the presence of cut propagators, which fix some loop energies without the need to use the residue theorem.

It remains to explain what we mean by step 4.b) and why it is always possible to achieve the proper diagrammatic form it refers to.

\subsection{Proper diagrammatic form and proper decomposition}

Before defining the proper diagrammatic form of the threshold decomposition, we illustrate the problem with two examples at one loop, level $\ell=1$ : the product $\Delta^{12} \mathcal{P}^{32} \mathcal{P}^{34}$, which does not have a proper diagrammatic form, and the product $\Delta^{12} \mathcal{P}^{32} \mathcal{P}^{14}$, which does. If we reinstate the loop energy $k^{0}$ leading to $\Delta^{12}$, we find

$$
\begin{aligned}
\Delta^{12} \mathcal{P}^{32} \mathcal{P}^{34} & =2 \pi^{2} \mathcal{P} \int \frac{\mathrm{d} k^{0}}{2 \pi} \delta\left(k^{0}-e_{1}+\omega_{1}\right) \delta\left(k^{0}-e_{2}-\omega_{2}\right) \frac{1}{e_{3}-k^{0}-\omega_{3}} \frac{1}{e_{3}-e_{4}-\omega_{3}-\omega_{4}} \\
\Delta^{12} \mathcal{P}^{32} \mathcal{P}^{14} & =2 \pi^{2} \mathcal{P} \int \frac{\mathrm{d} k^{0}}{2 \pi} \delta\left(k^{0}-e_{1}+\omega_{1}\right) \delta\left(k^{0}-e_{2}-\omega_{2}\right) \frac{1}{e_{3}-k^{0}-\omega_{3}} \frac{1}{k^{0}-e_{4}-\omega_{4}} .
\end{aligned}
$$

It is evident that the second line has a proper diagrammatic form, with the loop energy circulating in every factor. In the first line, the $\delta$ function of $\Delta^{12}$ cannot be used to modify $\mathcal{P}^{34}$. The reason is that the skeleton $\Delta^{12} \mathcal{P}^{32} \mathcal{P}^{34}$ has a non diagrammatic singularity, obtained by combining the singularities of $\Delta^{12}$ and $\mathcal{P}^{34}$ :

$$
e_{1}-e_{2}-\omega_{1}-\omega_{2}=e_{3}-e_{4}-\omega_{3}-\omega_{4}=0 .
$$

Such a singularity is not present in the skeletons we want to decompose, since they are originated by diagrams. This means that it must cancel with an opposite contribution coming from some other source. In section 5 we show that indeed it does.

The manipulations advocated in step 4.b) remove contributions like $\Delta^{12} \mathcal{P}^{32} \mathcal{P}^{34}$ in favor of contributions like $\Delta^{12} \mathcal{P}^{32} \mathcal{P}^{14}$. The importance of operation 4.b) will be appreciated starting from the box (section 5), since the triangle is too simple in this respect.

The proper form of the threshold decomposition (proper decomposition, from now on) of an $L$ loop connected diagram (prescribed à la Feynman everywhere) is the product of the proper decompositions of its 1PI ingredients. Vertices are considered 1PI irreducible subdiagrams. A propagator with no vertices attached to it is viewed as an 1PI subdiagram 
as well. The proper decomposition of a tree diagram follows from the diagrammatic rules of subsection 2.2 (every propagator being a link, in the sense explained there).

The proper decomposition of an $L$ loop 1PI diagram is defined by induction. Consider a 1PI skeleton diagram $\mathcal{G}_{L}^{s}$ with $L$ loops and $I$ internal legs. Let

$$
\mathcal{G}_{L}^{s}=\sum_{\ell=0}^{I-L} \mathcal{G}_{L, \ell}^{s}
$$

denote its threshold decomposition, organized into levels $\ell$. The $\ell=0$ contribution $\mathcal{G}_{L, 0}^{s}$ is the only one that truly has $L$ loops, because every $\mathcal{G}_{L, \ell}^{s}$ with $\ell \geqslant 1$ carries at least one $\delta$ function, which effectively breaks the skeleton by interrupting an internal leg and setting a condition on the external momenta. In the case of cut diagrams, the $\ell=0$ contribution $\mathcal{G}_{L, 0}^{s}$ is absent.

The proper decomposition of an $L$-loop cut diagram is straightforward, because a cut diagram is broken by the cuts into subdiagrams with smaller numbers of loops (whose proper decompositions are already known by the inductive assumption).

The proper decomposition of an uncut diagram is determined as follows. The decomposition of $\mathcal{G}_{L, \ell}^{s}$ is known from the optical theorem for every odd $\ell$, because it must match the decompositions of the cut diagrams. The decomposition of each $\mathcal{G}_{L, \ell}^{s}$ with even $\ell \geqslant 2$ is worked out from the one of $\mathcal{G}_{L, \ell-1}^{s}$ by circumventing the singularities associated with the principal values as demanded by the Feynman prescription (and paying attention to the combinatorics, to avoid overcounting). There is no ambiguity in this, since identities like (2.19) relate levels separated by an even number. Finally, the decomposition of $\mathcal{G}_{L, 0}^{s}$ is determined by subtraction.

The proper diagrammatic form of the zeroth level $\mathcal{G}_{L, 0}^{s}$ is not known a priori, but determined by the procedure itself. The reason is that the zeroth level is a sum of residues where every loop energy $k^{0}$ can have different values: several spurious thresholds and singularities (like the non diagrammatic ones and those due to the denominators $D_{\text {pseudo }}$ ) mutually cancel, although it is not manifest that they do.

The proper diagrammatic form of the threshold decomposition always exists and is unique. The skeleton singularities have a diagrammatic origin, so the non diagrammatic singularities can only appear in the intermediate steps, especially due to the manipulations of step 3), but must cancel out in the total. Moreover, the thresholds and singularities must appear with the right coefficients to match the diagram $G$ they are generated by. When that happens, the thresholds are properly extracted from the zeroth level to the levels $\ell \geqslant 1$ and the zeroth level defines the purely virtual content of the diagram $G$.

\section{Bubbles}

In this section we study the (multi)bubble diagrams. Although they do not present particular difficulties, they are a useful guide through the more complex configurations we study in the next sections.

The simple bubble diagram $\propto \alpha$ with physical particles propagating in the internal legs is denoted by (AB), or (A1B2). It gives the skeleton $B_{\mathrm{AB}}^{s}=G_{2}^{s}$ of formula (2.5). 
Integrating on the loop energy $k^{0}$ by means of the residue theorem, we obtain

$$
B_{\mathrm{AB}}^{s}=-i\left(F^{12}-F_{+-}^{12}\right)-i\left(F^{21}-F_{+-}^{21}\right),
$$

where $F_{+-}^{a b}=\left.F^{a b}\right|_{\omega_{a} \rightarrow-\omega_{a}}$. While $F^{12}$ and $F^{21}$ have the form $1 / \tilde{D}_{\text {phys }}, F_{+-}^{12}$ and $F_{+-}^{21}$ have

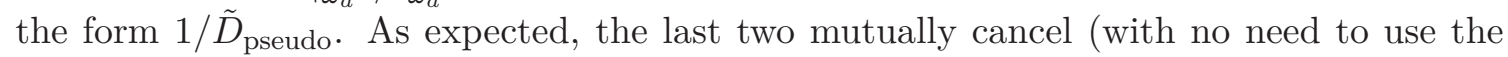
identities (2.21), in this case). In the end, we get

$$
B_{\mathrm{AB}}^{s}=-i\left(F^{12}+F^{21}\right)=-\frac{2 i\left(\omega_{1}+\omega_{2}\right)}{\left(e_{1}-e_{2}\right)^{2}-\left(\omega_{1}+\omega_{2}\right)^{2}+i \epsilon} .
$$

Using the decomposition (2.15) for $B_{\mathrm{AB}}^{s}$, which here reads

$$
F^{a b}=\mathcal{P}^{a b}-i \Delta^{a b}
$$

we find

$$
B_{\mathrm{AB}}^{s}=-i \hat{\mathcal{P}}^{12}-\Delta^{12}-\Delta^{21} .
$$

The conjugate diagram is $B_{\dot{\mathrm{A}} \mathrm{B}}^{s}=\bar{B}_{\mathrm{AB}}^{s}$. From the rules (2.12), the cut diagrams are $B_{\dot{\mathrm{AB}}}^{s}=2 \Delta^{21}$ and $B_{\mathrm{AB}}^{s}=2 \Delta^{12}$ (orienting $p_{1}$ from A to B). Collecting everything together, we obtain the table

\begin{tabular}{|c|c|c|c|c|}
\hline $\mathrm{Th} \backslash \mathrm{G}$ & $B_{\mathrm{AB}}^{s}$ & $B_{\dot{\mathrm{A}} \mathrm{B}}^{s}$ & $B_{\dot{\mathrm{A} B}}^{s}$ & $B_{\mathrm{AB}}^{s}$ \\
\hline- & $-i \hat{\mathcal{P}}^{12}$ & $i \hat{\mathcal{P}}^{12}$ & 0 & 0 \\
\hline$\Delta^{12}$ & -1 & -1 & 0 & 2 \\
\hline$\Delta^{21}$ & -1 & -1 & 2 & 0 \\
\hline
\end{tabular}

where "Th" stands for threshold and "G" stands for diagram. The thresholds are listed vertically, while the diagrams are listed horizontally.

Let $C_{i j}$ denote the entries of the table. A (cut or uncut) diagram $G_{j}$ is the $j$ th column of the table $(j>1)$, by which we mean the sum

$$
G_{j} \equiv \sum_{i>1} C_{i 1} C_{i j}
$$

where $C_{21}=1$. The spectral optical identities are "the rows of the table", by which we mean the identities

$$
R_{i} \equiv C_{i 1} \sum_{j>1} C_{i j}=0,
$$

for $i>1$. The spectral optical theorem

$$
B_{\mathrm{AB}}^{s}+B_{\dot{\mathrm{AB}}}^{s}+B_{\dot{\mathrm{AB}}}^{s}+B_{\mathrm{AB}}^{s}=0
$$

is the "sum of the entries" of (3.5), by which we mean the identity

$$
\sum_{j>1} G_{j}=\sum_{i>1} \sum_{j>1} C_{i 1} C_{i j}=0 .
$$

Finally, the optical theorem is the integral of this identity, multiplied by $4 \omega_{1} \omega_{2}$, over the space components of the loop momentum. 


\begin{tabular}{|c|c|c|c|c|c|c|c|c|}
\hline $\mathrm{Th} \backslash \mathrm{G}$ & $B_{\mathrm{ABC}}^{s}$ & $B_{\dot{\mathrm{A}} \dot{\mathrm{C}}}^{s}$ & $B_{\dot{\mathrm{A} B C}}^{s}$ & $B_{\mathrm{A} \dot{\mathrm{B} C}}^{s}$ & $B_{\mathrm{ABC}}^{s}$ & $B_{\mathrm{AB} \dot{\mathrm{C}}}^{s}$ & $B_{\dot{\mathrm{ABC}}}^{s}$ & $B_{\dot{\mathrm{A}} \dot{\mathrm{B} C}}^{s}$ \\
\hline- & $-i \hat{\mathcal{P}}^{12} \hat{\mathcal{P}}^{34}$ & $i \hat{\mathcal{P}}^{12} \hat{\mathcal{P}}^{34}$ & 0 & 0 & 0 & 0 & 0 & 0 \\
\hline$\Delta^{12}$ & $-\hat{\mathcal{P}}^{34}$ & $-\hat{\mathcal{P}}^{34}$ & 0 & 0 & 0 & $2 \hat{\mathcal{P}}^{34}$ & 0 & 0 \\
\hline$\Delta^{21}$ & $-\hat{\mathcal{P}}^{34}$ & $-\hat{\mathcal{P}}^{34}$ & $2 \hat{\mathcal{P}}^{34}$ & 0 & 0 & 0 & 0 & 0 \\
\hline$\Delta^{34}$ & $-\hat{\mathcal{P}}^{12}$ & $-\hat{\mathcal{P}}^{12}$ & 0 & 0 & $2 \hat{\mathcal{P}}^{12}$ & 0 & 0 & 0 \\
\hline$\Delta^{43}$ & $-\hat{\mathcal{P}}^{12}$ & $-\hat{\mathcal{P}}^{12}$ & 0 & 0 & 0 & 0 & 0 & $2 \hat{\mathcal{P}}^{12}$ \\
\hline$\Delta^{12} \Delta^{34}$ & $i$ & $-i$ & 0 & 0 & $-2 i$ & $2 i$ & 0 & 0 \\
\hline$\Delta^{12} \Delta^{43}$ & $i$ & $-i$ & 0 & $-4 i$ & 0 & $2 i$ & 0 & $2 i$ \\
\hline$\Delta^{21} \Delta^{34}$ & $i$ & $-i$ & $-2 i$ & 0 & $-2 i$ & 0 & $4 i$ & 0 \\
\hline$\Delta^{21} \Delta^{43}$ & $i$ & $-i$ & $-2 i$ & 0 & 0 & 0 & 0 & $2 i$ \\
\hline
\end{tabular}

Table 1. Threshold decomposition of the double bubble.

With a fakeon in either internal leg, or in both, we denote the diagram by (AfB), (AfBf), etc., depending on the case. We need to apply step 5) of subsection 2.5, which amounts to drop the thresholds identified by $\Delta^{12}$ and $\Delta^{21}$, since each of them contains at least one fakeon frequency. The table loses the last two rows, so we obtain

$$
B_{\mathrm{AfB}}^{s}=B_{\mathrm{AfBf}}^{s}=-i \hat{\mathcal{P}}^{12}, \quad B_{\dot{\mathrm{AfB}}}^{s}=B_{\dot{\mathrm{AfBf}}}^{s}=i \hat{\mathcal{P}}^{12} .
$$

No nontrivial cut diagram survives, in agreement with the rules of subsection 2.2.

The double bubble $\mathrm{OO} \alpha$ is $(\mathrm{AB})(\mathrm{BC})$, or (A1B2)(B3C4). Without the risk of confusion, we just call it $\mathrm{ABC}$ in table 1, where we show its threshold decomposition.

With a fakeon in leg 1 , we drop $\Delta^{12}$ and $\Delta^{21}$ everywhere and obtain

\begin{tabular}{|c|c|c|c|c|}
\hline Th $\backslash \mathrm{G}$ & $B_{\mathrm{AfBC}}^{s}$ & $B_{\dot{\mathrm{A} f \mathrm{~B} \dot{C}}}^{s}$ & $B_{\text {AfBC }}^{s}$ & $B_{\text {AfBC }}^{s}$ \\
\hline- & $-i \hat{\mathcal{P}}^{12} \hat{\mathcal{P}}^{34}$ & $i \hat{\mathcal{P}}^{12} \hat{\mathcal{P}}^{34}$ & 0 & 0 \\
\hline$\Delta^{34}$ & $-\hat{\mathcal{P}}^{12}$ & $-\hat{\mathcal{P}}^{12}$ & $2 \hat{\mathcal{P}}^{12}$ & 0 \\
\hline$\Delta^{43}$ & $-\hat{\mathcal{P}}^{12}$ & $-\hat{\mathcal{P}}^{12}$ & 0 & $2 \hat{\mathcal{P}}^{12}$ \\
\hline
\end{tabular}

With fakeons in legs 1 and 2, we get the same. With a fakeon in leg 1 and one in leg 3 , we obtain

$$
B_{\mathrm{AfBfC}}^{s}=-i \hat{\mathcal{P}}^{12} \hat{\mathcal{P}}^{34}, \quad B_{\text {AfBfC }}^{s}=i \hat{\mathcal{P}}^{12} \hat{\mathcal{P}}^{34}
$$

We can repeat the derivation for the skeletons of multibubble diagrams 1000010 . The result is the product of the skeletons of the single bubbles, for all choices of physical particles and fakeons.

In all cases, the sum (3.6) of each column equals the (cut or uncut) skeleton diagram shown at the top of it. Moreover, the spectral optical identities are the sums (3.7) of the rows. The spectral optical theorem is obtained by summing all the entries of the table as 
in (3.9). For example, for the double bubble diagram $\backslash O O \alpha$ we have

$$
\begin{aligned}
& B_{\mathrm{ABC}}^{s}+B_{\dot{\mathrm{A}} \dot{\mathrm{BC}}}^{s}+B_{\dot{\mathrm{ABC}}}^{s}+B_{\mathrm{ABC}}^{s}+B_{\mathrm{ABC}}^{s}+B_{\mathrm{AB} \dot{\mathrm{C}}}^{s}+B_{\dot{\mathrm{ABC}}}^{s}+B_{\dot{\mathrm{A}} \dot{\mathrm{BC}}}^{s}=0 \text {, with no fakeons, } \\
& B_{\mathrm{AfBC}}^{s}+B_{\dot{\mathrm{A} f B \dot{C}}}^{s}+B_{\mathrm{AfBC}}^{s}+B_{\dot{\mathrm{A} f \dot{ } \mathrm{C}}}^{s}=0 \text {, with a fakeon in } \mathrm{AB} \text {, } \\
& B_{\mathrm{AfBfC}}^{s}+B_{\dot{\mathrm{AfBfC}}}^{s}=0, \quad \text { with a fakeon in } \mathrm{AB} \\
& \text { and one in BC. }
\end{aligned}
$$

The usual optical theorem is obtained by integrating the spectral optical theorem (divided by $2 \omega$ for every internal leg) over the space components of the loop momentum or the phase spaces (in cut diagrams). For example, with a fakeon in $\mathrm{AB}$, we obtain

$$
B_{\mathrm{AfBC}}+B_{\dot{\mathrm{AfB} \dot{\mathrm{C}}}}+B_{\mathrm{AfB} \dot{\mathrm{C}}}+B_{\dot{\mathrm{AfBC}}}=0 .
$$

The final outcome is that the single bubble diagram with one or two internal fakeons is equal to $i$ times the imaginary part of the usual bubble diagram:

$$
X^{\mathrm{f}}=\chi_{\mathrm{f}}^{\mathrm{f}} X=i \operatorname{Im}[X] \text {. }
$$

The $n$th multibubble diagram is the product of the single bubble diagrams it is made of, times $i^{n-1}$. It is easy to check that the diagrammatics of cut and uncut diagrams, with or without fakeons, agrees with the one stated in subsection 2.2.

\section{Triangle}

In this section we study the triangle diagram, denoted by (ABC), or (A1B2C3). Its skeleton is $T_{\mathrm{ABC}}^{s}=G_{3}^{s}$, from formula (2.5). For convenience, we integrate on the loop energy $k^{0}$ by averaging on the two ways to close the integration path at infinity and using the residue theorem. Without using common denominators or other manipulations, the result can be expanded as

$$
T_{\mathrm{ABC}}^{s}=-i F_{\mathrm{ABC}}-i F_{1 \mathrm{ABC}}-i F_{2 \mathrm{ABC}}
$$

where

$$
\begin{aligned}
F_{\mathrm{ABC}} & =F^{12} F^{13}+\operatorname{cycl}+(e \rightarrow-e), \\
F_{1 \mathrm{ABC}} & =\frac{1}{2} S^{12 \mid 13}+\operatorname{cycl}+(e \rightarrow-e), \quad F_{2 \mathrm{ABC}}=-\frac{1}{2} S_{++}^{12 \mid 31}+(e \rightarrow-e), \\
S^{a b \mid c d} & =S\left(e_{a}-e_{b}-\tilde{\omega}_{a}-\tilde{\omega}_{b},-e_{c}+e_{d}+\tilde{\omega}_{c}+\tilde{\omega}_{d}\right), \quad \hat{S}^{a b \mid c d}=\left.S^{a b \mid d c}\right|_{\tilde{\omega}_{b} \rightarrow-\tilde{\omega}_{b}, \tilde{\omega}_{c} \rightarrow-\tilde{\omega}_{c}} .
\end{aligned}
$$

Here, "cycl" refers to the cyclic permutations of 1,2 and 3 and $S(x, y)$ is defined in (2.16).

Because of identities like (2.17), both $S^{a b \mid c d}$ and $\hat{S}^{a b \mid c d}$ vanish. This is where the operation (2.21) eliminates all the pseudothresholds and leaves the physical thresholds only. We obtain

$$
T_{\mathrm{ABC}}^{s}=-i F_{\mathrm{ABC}}
$$




\begin{tabular}{|c|c|c|c|c|c|c|c|c|}
\hline $\mathrm{Th} \backslash \mathrm{G}$ & $T_{\mathrm{ABC}}^{s}$ & $T_{\dot{\mathrm{A}} \dot{\mathrm{B}}}^{s}$ & $T_{\dot{\mathrm{ABC}}}^{s}$ & $T_{\mathrm{ABC}}^{s}$ & $T_{\mathrm{ABC}}^{s}$ & $T_{\mathrm{AB} \dot{\mathrm{C}}}^{s}$ & $T_{\mathrm{ABC}}^{s}$ & $T_{\dot{\mathrm{A}} \dot{ } \mathrm{C}}^{s}$ \\
\hline- & $-i \mathcal{P}_{\mathrm{ABC}}$ & $i \mathcal{P}_{\mathrm{ABC}}$ & 0 & 0 & 0 & 0 & 0 & 0 \\
\hline$\Delta^{23}$ & $-\mathcal{Q}^{21}$ & $-\mathcal{Q}^{21}$ & 0 & 0 & 0 & 0 & 0 & $2 \mathcal{Q}^{21}$ \\
\hline$\Delta^{12}$ & $-\mathcal{Q}^{13}$ & $-\mathcal{Q}^{13}$ & 0 & 0 & 0 & 0 & $2 \mathcal{Q}^{13}$ & 0 \\
\hline$\Delta^{31}$ & $-\mathcal{Q}^{32}$ & $-\mathcal{Q}^{32}$ & 0 & 0 & 0 & $2 \mathcal{Q}^{32}$ & 0 & 0 \\
\hline$\Delta^{32}$ & $-\mathcal{Q}^{31}$ & $-\mathcal{Q}^{31}$ & 0 & 0 & $2 \mathcal{Q}^{31}$ & 0 & 0 & 0 \\
\hline$\Delta^{21}$ & $-\mathcal{Q}^{23}$ & $-\mathcal{Q}^{23}$ & 0 & $2 \mathcal{Q}^{23}$ & 0 & 0 & 0 & 0 \\
\hline$\Delta^{13}$ & $-\mathcal{Q}^{12}$ & $-\mathcal{Q}^{12}$ & $2 \mathcal{Q}^{12}$ & 0 & 0 & 0 & 0 & 0 \\
\hline$\Delta^{12} \Delta^{13}$ & $i$ & $-i$ & $-2 i$ & 0 & 0 & 0 & $2 i$ & 0 \\
\hline$\Delta^{23} \Delta^{21}$ & $i$ & $-i$ & 0 & $-2 i$ & 0 & 0 & 0 & $2 i$ \\
\hline$\Delta^{31} \Delta^{32}$ & $i$ & $-i$ & 0 & 0 & $-2 i$ & $2 i$ & 0 & 0 \\
\hline$\Delta^{31} \Delta^{21}$ & $i$ & $-i$ & 0 & $-2 i$ & 0 & $2 i$ & 0 & 0 \\
\hline$\Delta^{12} \Delta^{32}$ & $i$ & $-i$ & 0 & 0 & $-2 i$ & 0 & $2 i$ & 0 \\
\hline$\Delta^{23} \Delta^{13}$ & $i$ & $-i$ & $-2 i$ & 0 & 0 & 0 & 0 & $2 i$ \\
\hline
\end{tabular}

Table 2. Threshold decomposition of the triangle.

With the help of (2.15), or (3.3), the threshold decomposition gives

$$
T_{\mathrm{ABC}}^{s}=-i \mathcal{P}_{\mathrm{ABC}}-\sum_{\text {perms }} \Delta^{a b} \mathcal{Q}^{a c}+\frac{i}{2} \sum_{\text {perms }} \Delta^{a b}\left(\Delta^{a c}+\Delta^{c b}\right),
$$

where $\mathcal{P}_{\mathrm{ABC}}=\left.F_{\mathrm{ABC}}\right|_{F \rightarrow \mathcal{P}}$ and the sums are on $\{a, b, c\}$ equal to the permutations of 1 , 2 and 3. The conjugate diagram is $T_{\dot{\mathrm{A}} \dot{\mathrm{C}}}^{s}=\bar{T}_{\mathrm{ABC}}^{s}$. The cut diagrams follow from the diagrammatic rules of subsection 2.2. Orienting $p_{1}$ from $\mathrm{B}$ to $\mathrm{A}$, we have

$$
T_{\mathrm{ABC}}^{s}=2 \Delta^{21}\left(\mathcal{Q}^{23}-i \Delta^{31}-i \Delta^{23}\right), \quad T_{\dot{\mathrm{AB}} \dot{\mathrm{C}}}^{s}=2 \Delta^{12}\left(\mathcal{Q}^{13}+i \Delta^{13}+i \Delta^{32}\right),
$$

the other ones being derived by cyclically permuting 1,2 and 3 . In the end, we obtain table 2. The diagrams are given by the columns, as per (3.6).

The entries of the table are organized in a diagrammatic form, as per point 4.b) of subsection 2.5. Indeed,

$$
\Delta^{a b} \mathcal{Q}^{a c}=\Delta^{a b} \mathcal{Q}^{c b}=16 \pi^{2} \omega_{a} \omega_{b} \omega_{c} \mathcal{P} \int \frac{\mathrm{d} k^{0}}{2 \pi} \frac{\delta^{-}\left(\left(k-p_{a}\right)^{2}-m_{a}^{2}\right) \delta^{+}\left(\left(k-p_{b}\right)^{2}-m_{b}^{2}\right)}{\left(k-p_{c}\right)^{2}-m_{c}^{2}},
$$

where $\delta^{ \pm}\left(p^{2}-m^{2}\right)=\theta\left( \pm p^{0}\right) \delta\left(p^{2}-m^{2}\right)$. As soon as we divide by $8 \omega_{a} \omega_{b} \omega_{c}$ and integrate on the space components of the loop momentum, the result is Lorentz invariant.

The spectral optical identities are the rows (3.7) of table 2, which vanish separately. The spectral optical theorem is obtained by summing all the entries of the table as in (3.9):

$$
T_{\mathrm{ABC}}^{s}+T_{\dot{\mathrm{A}} \dot{\mathrm{B}} \dot{\mathrm{C}}}^{s}+T_{\dot{\mathrm{ABC}}}^{s}+T_{\mathrm{ABC}}^{s}+T_{\mathrm{ABC}}^{s}+T_{\mathrm{AB} \dot{\mathrm{C}}}^{s}+T_{\mathrm{ABC}}^{s}+T_{\dot{\mathrm{ABC}}}^{s}=0 .
$$


The usual optical theorem

$$
T_{\mathrm{ABC}}+T_{\dot{\mathrm{AB}} \dot{\mathrm{C}}}+T_{\dot{\mathrm{ABC}}}+T_{\mathrm{ABC}}+T_{\mathrm{ABC}}+T_{\mathrm{AB \dot {C }}}+T_{\dot{\mathrm{AB}} \dot{\mathrm{C}}}+T_{\dot{\mathrm{ABC}}}=0
$$

is obtained dividing (4.7) by $8 \omega_{1} \omega_{2} \omega_{3}$ and integrating on the space components $\mathbf{k}$ of the loop momentum. It is also evident that (4.7) and (4.8) agree with the diagrammatic rules of subsection 2.2 .

\subsection{Fakeons}

Now we study the fakeon prescription/projection. Assume that leg 1 , which is the segment $\mathrm{AB}$, is a fakeon and the other two internal legs are physical particles. Then $\omega_{1}$ is a fakeon frequency. According to step 5) of subsection 2.5, we must suppress all the thresholds involving $\omega_{1}$, i.e., the single thresholds proportional to $\Delta^{12}, \Delta^{21}, \Delta^{13}$ and $\Delta^{31}$, and all the double thresholds. So doing, we obtain

$$
\begin{array}{ll}
T_{\mathrm{AfBC}}^{s}=-i \mathcal{P}_{\mathrm{ABC}}-\Delta^{23} \mathcal{Q}^{21}-\Delta^{32} \mathcal{Q}^{31}, & T_{\mathrm{AfBC}}^{s}=2 \Delta^{32} \mathcal{Q}^{31}, \\
T_{\mathrm{A} \dot{\mathrm{ABC}}}^{s}=i \mathcal{P}_{\mathrm{ABC}}-\Delta^{23} \mathcal{Q}^{21}-\Delta^{32} \mathcal{Q}^{31}, & T_{\mathrm{AfBC}}^{s}=2 \Delta^{23} \mathcal{Q}^{21},
\end{array}
$$

and the table

\begin{tabular}{|c|c|c|c|c|}
\hline $\mathrm{Th} \backslash \mathrm{G}$ & $T_{\mathrm{AfBC}}^{s}$ & $T_{\dot{\mathrm{A} f B C}}^{s}$ & $T_{\mathrm{AfBC}}^{s}$ & $T_{\text {AfB் }}^{s}$ \\
\hline- & $-i \mathcal{P}_{\mathrm{ABC}}$ & $i \mathcal{P}_{\mathrm{ABC}}$ & 0 & 0 \\
\hline$\Delta^{23}$ & $-\mathcal{Q}^{21}$ & $-\mathcal{Q}^{21}$ & 0 & $2 \mathcal{Q}^{21}$ \\
\hline$\Delta^{32}$ & $-\mathcal{Q}^{31}$ & $-\mathcal{Q}^{31}$ & $2 \mathcal{Q}^{31}$ & 0 \\
\hline
\end{tabular}

As usual, the diagrams are the columns (3.6) of the table and the spectral optical identities are the rows (3.7). The spectral optical theorem and the ordinary optical theorem are the sums

$$
\begin{aligned}
& T_{\mathrm{AfBC}}^{s}+T_{\dot{\mathrm{A} f \dot{B} \dot{C}}}^{s}+T_{\mathrm{AfBC}}^{s}+T_{\dot{\mathrm{AfBC}}}^{s}=0, \\
& T_{\mathrm{AfBC}}+T_{\dot{\mathrm{A} f \dot{B} \dot{C}}}+T_{\mathrm{AfBC}}+T_{\dot{\mathrm{AfB} C}}=0 .
\end{aligned}
$$

Again, these identities agree with the diagrammatic rules of section 2.2. In particular, since the shadowed and unshadowed portions of the cut triangle are tree diagrams, the fakeon propagator in $\mathrm{AB}$ is a link in $T_{\mathrm{AfBC}}^{s}$ and $T_{\dot{\mathrm{AfB} C} \mathrm{C}}^{s}$, so it is given by (2.14).

With two or three fakeons, we drop all the thresholds and obtain

$$
T_{\mathrm{AfBfC}}^{s}=T_{\mathrm{AfBfCf}}^{s}=-i \mathcal{P}_{\mathrm{ABC}}=i \operatorname{Im}\left[T_{\mathrm{ABC}}^{s}\right]-\frac{i}{2} \sum_{\text {perms }} \Delta^{a b}\left(\Delta^{a c}+\Delta^{c b}\right) .
$$

In this case, the skeleton diagram is purely imaginary, so no cut diagrams survive. The result (4.12) encodes the purely virtual content of the triangle.

Formulas (4.9) and (4.12) can be used to relate the triangles with fakeons to the standard triangle, for possible implementations in softwares like FeynCalc, FormCalc, LoopTools and Package-X [26-32]. 


\section{Box}

In this section we study the box diagram $G_{4}$, denoted by (ABCD), or (A1B2C3D4). We consider its skeleton $G_{4}^{s}$ and integrate on the loop energy $k^{0}$. Then, we use smart identities (2.21) to make the pseudothresholds disappear, which we know to be possible because they are not physical. The result of these operations is

$$
G_{\mathrm{ABCD}}^{s 4}=-\frac{i}{6} \sum_{\text {perms }} F^{a b} F^{a c} F^{a d}-\frac{i}{4} \sum_{\text {perms }} F^{a b} F^{a c} F^{d b}+(e \rightarrow-e),
$$

where the sums are evaluated for $\{a, b, c, d\}$ ranging over the set of permutations of $1,2,3$ and 4 .

To derive the threshold decomposition, we first apply (2.15), or (3.3), and expand. The terms proportional one $\delta$ function, which are

$$
-\frac{1}{2} \sum_{\text {perms }} \Delta^{a b} \mathcal{P}^{a c} \mathcal{P}^{a d}-\frac{1}{4} \sum_{\text {perms }}\left(\Delta^{a b} \mathcal{P}^{a c} \mathcal{P}^{d b}+\mathcal{P}^{a b} \Delta^{a c} \mathcal{P}^{d b}+\mathcal{P}^{a b} \mathcal{P}^{a c} \Delta^{d b}\right)
$$

plus $(e \rightarrow-e)$, mix single thresholds and triple thresholds, due to identities like (2.19). We can separate the two types of contributions by fulfilling the proper diagrammatic requirement 4.b) of subsection 2.5. Consider, for example, the terms proportional to $\Delta^{12}$ (all the others being treated the same way), which read

$$
\begin{gathered}
w_{12} \equiv-\Delta^{12}\left[\mathcal{P}^{13} \mathcal{P}^{14}+\mathcal{P}^{32} \mathcal{P}^{42}+\frac{1}{2}\left(\mathcal{P}^{14} \mathcal{P}^{32}+\mathcal{P}^{14} \mathcal{P}^{34}+\mathcal{P}^{32} \mathcal{P}^{34}\right)\right. \\
\left.+\frac{1}{2}\left(\mathcal{P}^{13} \mathcal{P}^{42}+\mathcal{P}^{13} \mathcal{P}^{43}+\mathcal{P}^{42} \mathcal{P}^{43}\right)\right]
\end{gathered}
$$

Even if we restrict the sum between the square brackets to the support of the $\delta$ function, we can easily check that the expression does not have a proper diagrammatic form, as in the first line of $(2.23)$, because $\mathcal{P}^{34}$ and $\mathcal{P}^{43}$ are unaffected by $\Delta^{12}$. We can adjust $w_{12}$ by adding and subtracting

$$
\tilde{w}_{12} \equiv-\Delta^{12}\left[\frac{1}{2}\left(\mathcal{P}^{14} \mathcal{P}^{32}-\mathcal{P}^{14} \mathcal{P}^{34}-\mathcal{P}^{32} \mathcal{P}^{34}\right)+\frac{1}{2}\left(\mathcal{P}^{13} \mathcal{P}^{42}-\mathcal{P}^{13} \mathcal{P}^{43}-\mathcal{P}^{42} \mathcal{P}^{43}\right)\right] .
$$

Indeed, the sum

$$
\begin{aligned}
w_{12}+\tilde{w}_{12} & =-\Delta^{12}\left(\mathcal{P}^{13} \mathcal{P}^{14}+\mathcal{P}^{32} \mathcal{P}^{42}+\mathcal{P}^{14} \mathcal{P}^{32}+\mathcal{P}^{13} \mathcal{P}^{42}\right)=-\Delta^{12} \mathcal{Q}^{13} \mathcal{Q}^{14} \\
& =-32 \pi^{2} \omega_{1} \omega_{2} \omega_{3} \omega_{4} \mathcal{P} \int \frac{\mathrm{d} k^{0}}{2 \pi} \frac{\delta^{-}\left(\left(k-p_{1}\right)^{2}-m_{1}^{2}\right)}{\left(k-p_{3}\right)^{2}-m_{3}^{2}} \frac{\delta^{+}\left(\left(k-p_{2}\right)^{2}-m_{2}^{2}\right)}{\left(k-p_{4}\right)^{2}-m_{4}^{2}}
\end{aligned}
$$

has a proper diagrammatic form. On the other hand, it is easy to show, by means of the first identity of formula (2.19), that the contribution we need to subtract is a triple threshold:

$$
\tilde{w}_{12}=\frac{1}{2} \Delta^{12} \Delta^{32} \Delta^{14}+\frac{1}{2} \Delta^{12} \Delta^{42} \Delta^{13} .
$$

Therefore, we move it down to level 3. 
The terms proportional to the product of two $\delta$ functions in (5.1), i.e.,

$$
\frac{i}{2} \sum_{\text {perms }} \Delta^{a b} \Delta^{a c} \mathcal{P}^{a d}+\frac{i}{4} \sum_{\text {perms }}\left(\Delta^{a b} \Delta^{a c} \mathcal{P}^{d b}+\mathcal{P}^{a b} \Delta^{a c} \Delta^{d b}+\Delta^{a b} \mathcal{P}^{a c} \Delta^{d b}\right)
$$

plus $(e \rightarrow-e)$, are all double thresholds, since there is no product of principal values here that needs to be rearranged. For example, the terms proportional to $\Delta^{12} \Delta^{13}$ and $\Delta^{12} \Delta^{34}$ (the other possibilities being simple transformations of these), either vanish or can be readily written in a proper diagrammatic form:

$$
i \Delta^{12} \Delta^{13}\left(\mathcal{P}^{14}+\frac{1}{2} \mathcal{P}^{42}+\frac{1}{2} \mathcal{P}^{43}\right)=i \Delta^{12} \Delta^{13} \mathcal{Q}^{14}, \quad \frac{i}{2} \Delta^{12} \Delta^{34}\left(\mathcal{P}^{14}+\mathcal{P}^{32}\right)=0 .
$$

Finally, the triple thresholds are $\left.G_{\mathrm{ABCD}}^{s 4}\right|_{F \rightarrow-i \Delta}$, from formula (5.1), plus the contributions like (5.6) coming down from the terms proportional to one $\delta$ function.

\subsection{Formulas}

In the end, the threshold decomposition of the box skeleton diagram reads

$$
\begin{aligned}
G_{\mathrm{ABCD}}^{s 4}= & -i \mathcal{P}_{4}-\frac{1}{2} \sum_{\text {perms }} \Delta^{a b} \mathcal{Q}^{a c} \mathcal{Q}^{a d}+\frac{i}{2} \sum_{\text {perms }} \Delta^{a b}\left(\Delta^{a c}+\Delta^{c b}\right) \mathcal{Q}^{a d} \\
& +\frac{1}{6} \sum_{\text {perms }} \Delta^{a b}\left(\Delta^{a c} \Delta^{a d}+\Delta^{c b} \Delta^{d b}\right)
\end{aligned}
$$

where $\mathcal{P}_{4}=\left.i G_{\mathrm{ABCD}}^{s 4}\right|_{F \rightarrow \mathcal{P}}$. We obtain the table

\begin{tabular}{|c|c|c|c|c|c|c|}
\hline $\mathrm{Th} \backslash \mathrm{G}$ & $G_{\mathrm{ABCD}}^{s 4}$ & $G_{\dot{\mathrm{A} \dot{\mathrm{B}} \mathrm{C}}}^{s 4}$ & {$\left[G_{\mathrm{ABCD}}^{s 4}\right.$} & $G_{\dot{\mathrm{AB}} \mathrm{C} \dot{\mathrm{D}}}^{s 4}$ & $G_{\dot{\mathrm{ABCD}}}^{s 4}$ & $\left.\frac{1}{2} G_{\dot{\mathrm{A} B \dot{C} \mathrm{D}}}^{s 4}\right]$ \\
\hline- & $-i \mathcal{P}_{4}$ & $i \mathcal{P}_{4}$ & 0 & 0 & 0 & 0 \\
\hline$\left[\Delta^{12}\right]$ & $-\mathcal{Q}^{13} \mathcal{Q}^{14}$ & $-\mathcal{Q}^{13} \mathcal{Q}^{14}$ & 0 & $2 \mathcal{Q}^{13} \mathcal{Q}^{14}$ & 0 & 0 \\
\hline$\left[\Delta^{21}\right]$ & $-\mathcal{Q}^{23} \mathcal{Q}^{24}$ & $-\mathcal{Q}^{23} \mathcal{Q}^{24}$ & $2 \mathcal{Q}^{23} \mathcal{Q}^{24}$ & 0 & 0 & 0 \\
\hline$\left[\Delta^{13}\right]$ & $-\mathcal{Q}^{12} \mathcal{Q}^{14}$ & $-\mathcal{Q}^{12} \mathcal{Q}^{14}$ & 0 & 0 & $2 \mathcal{Q}^{12} \mathcal{Q}^{14}$ & 0 \\
\hline$\ldots$ & $\ldots$ & $\ldots$ & $\ldots$ & $\ldots$ & $\ldots$ & $\ldots$ \\
\hline
\end{tabular}

where we have reported only the single thresholds. The contributions between brackets must be summed over the cyclic permutations of $1,2,3,4$.

The complex conjugate diagram is $G_{\dot{\mathrm{A}} \dot{\mathrm{B}} \dot{\mathrm{D}}}^{s 4}=\bar{G}_{\mathrm{ABCD}}^{s 4}$. The decompositions of the cut diagrams are

$$
\begin{aligned}
& G_{\dot{\mathrm{ABC}} \dot{\mathrm{C}}}^{s 4}=2 \Delta^{12} \mathcal{Q}^{13} \mathcal{Q}^{14}+2 i \Delta^{12}\left[\Delta^{13} \mathcal{Q}^{14}+\Delta^{14} \mathcal{Q}^{13}+\Delta^{32} \mathcal{Q}^{14}+\Delta^{42} \mathcal{Q}^{13}\right] \\
& -2 \Delta^{12}\left[\Delta^{13} \Delta^{14}+\Delta^{14} \Delta^{34}+\Delta^{13} \Delta^{43}+\Delta^{32} \Delta^{42}\right], \\
& G_{\dot{\mathrm{ABCD}}}^{s 4}=2 \Delta^{13} \mathcal{Q}^{12} \mathcal{Q}^{14}+2 i \Delta^{13}\left[\Delta^{14} \mathcal{Q}^{12}+\Delta^{43} \mathcal{Q}^{12}-\Delta^{23} \mathcal{Q}^{14}-\Delta^{12} \mathcal{Q}^{14}\right] \\
& +2 \Delta^{13}\left[\Delta^{12} \Delta^{14}+\Delta^{14} \Delta^{24}+\Delta^{23} \Delta^{43}+\Delta^{42} \Delta^{43}\right], \\
& G_{\mathrm{ABCD}}^{s 4}=\left.\bar{G}_{\dot{\mathrm{ABC}} \dot{\mathrm{D}}}^{s 4}\right|_{e \rightarrow-e}, \quad G_{\dot{\mathrm{ABCD}}}^{s 4}=8 \Delta^{12} \Delta^{14} \Delta^{34},
\end{aligned}
$$

up to cyclic permutations. 
Using (5.9) and (5.11), it is easy to check the spectral optical theorem

$$
G_{\mathrm{ABCD}}^{s 4}+G_{\dot{\mathrm{A} \dot{B} \dot{C} \dot{D}}}^{s 4}+\left[G_{\mathrm{ABCD}}^{s 4}+G_{\dot{\mathrm{AB}} \dot{\mathrm{C} \dot{D}}}^{s 4}+G_{\dot{\mathrm{ABCD}}}^{s 4}+\frac{1}{2} G_{\dot{\mathrm{AB}} \dot{\mathrm{C} D}}^{s 4}+\mathrm{cycl}\right]=0 .
$$

The factor $1 / 2$ avoids overcounting when the cyclic permutations are included. As usual, the optical theorem

$$
G_{\mathrm{ABCD}}^{4}+G_{\dot{\mathrm{A} \dot{\mathrm{B}} \dot{\mathrm{D}}}}^{4}+\left[G_{\mathrm{A} \dot{\mathrm{B} C D}}^{4}+G_{\dot{\mathrm{A} B \dot{\mathrm{C}} \dot{\mathrm{D}}}}^{4}+G_{\dot{\mathrm{A} B C \dot{D}}}^{4}+\frac{1}{2} G_{\dot{\mathrm{AB}} \dot{\mathrm{C} D}}^{4}+\mathrm{cycl}\right]=0
$$

is obtained by integrating on the space components of the loop momentum (after dividing by $2 \omega$ for every internal leg). Finally, the spectral optical identities are obtained by separating the contributions of the various (multi)thresholds to (5.12).

\subsection{Fakeons}

When fakeons are present, we apply the fakeon prescription/projection mentioned in step 5) of subsection 2.5, which amounts to eliminating the contributions coming from the thresholds that involve one or more fakeons. The spectral optical identities and the spectral optical theorem continue to hold after these operations, since the thresholds do not interfere with one another.

Concretely, with one fakeon in leg 4 , we must suppress all the $\Delta^{a b}$ with $a$ or $b$ equal to 4 . From (5.9), the uncut diagram is given by

$$
G_{\mathrm{ABCDf}}^{s 4}=-i \mathcal{P}_{4}-\sum_{p(1,2,3)} \Delta^{a b} \mathcal{Q}^{a c} \mathcal{Q}^{a 4}+\frac{i}{2} \sum_{p(1,2,3)} \Delta^{a b}\left(\Delta^{a c}+\Delta^{c b}\right) \mathcal{Q}^{a 4},
$$

where the sum is restricted to the permutations $\{a, b, c\}$ of 1,2 and 3 . The nonvanishing cut diagrams are

$$
\begin{aligned}
& G_{\mathrm{ABCDf}}^{s 4}=\left.\bar{G}_{\dot{\mathrm{ABC}} \mathrm{C} \dot{\mathrm{Df}}}^{s 4}\right|_{e \rightarrow-e}, \quad G_{\dot{\mathrm{AB}} \mathrm{C} \dot{\mathrm{Df}}}^{s 4}=2 \Delta^{12} \mathcal{Q}^{14}\left(\mathcal{Q}^{13}+i \Delta^{13}+i \Delta^{32}\right), \\
& G_{\mathrm{ABC \dot {C } \mathrm { f }}}^{s 4}=\bar{G}_{\left.\dot{\mathrm{A} \dot{B} C \dot{D} \mathrm{f}}\right|_{e \rightarrow-e}}, \quad G_{\dot{\mathrm{A} \dot{\mathrm{C}} \mathrm{C} \dot{f}}}^{s 4}=2 \Delta^{23} \mathcal{Q}^{24}\left(\mathcal{Q}^{21}+i \Delta^{21}+i \Delta^{13}\right), \\
& G_{\dot{\mathrm{ABCD} f}}^{s 4}=2 \Delta^{13} \mathcal{Q}^{14}\left(\mathcal{Q}^{12}-i \Delta^{23}-i \Delta^{12}\right), \quad G_{\mathrm{AB \dot {C }} \mathrm{Df}}^{s 4}=\left.\bar{G}_{\dot{\mathrm{ABCDf}}}^{s 4}\right|_{e \rightarrow-e} .
\end{aligned}
$$

It is easy to verify that these expressions satisfy the spectral optical theorem

$$
G_{\mathrm{ABCDf}}^{s 4}+G_{\dot{\mathrm{A} \dot{\mathrm{B}} \mathrm{D} \dot{f}}}^{s 4}+G_{\mathrm{A \dot {B } C D f}}^{s 4}+G_{\dot{\mathrm{ABC}} \dot{\mathrm{Df}}}^{s 4}+G_{\mathrm{ABC} \mathrm{Df}}^{s 4}+G_{\dot{\mathrm{A} \dot{\mathrm{B} C} \dot{ } \mathrm{f}}}^{s 4}+G_{\dot{\mathrm{ABCDf}}}^{s 4}+G_{\mathrm{A \dot {B }} \dot{\mathrm{C} D f}}^{s 4}=0,
$$

where $G_{\dot{\mathrm{A}} \dot{\mathrm{B}} \dot{\mathrm{C}} \mathrm{Df}}^{s 4}=\bar{G}_{\mathrm{ABCDf}}^{s 4}$, and match the diagrammatics of section 2.2. The vanishing cut diagrams are those that contain one cut fakeon propagator, which is identically zero by (2.13), so they also agree with the diagrammatics.

With fakeons in 3 and 4 we have

$$
G_{\mathrm{ABCfDf}}^{s 4}=-i \mathcal{P}_{4}-\Delta^{12} \mathcal{Q}^{13} \mathcal{Q}^{14}-\Delta^{21} \mathcal{Q}^{23} \mathcal{Q}^{24},
$$

the nonvanishing cut diagrams being

$$
G_{\mathrm{AB \dot {CfDf }}}^{s 4}=\left.\bar{G}_{\dot{\mathrm{ABC}} \mathrm{C} \dot{\mathrm{D} f}}\right|_{e \rightarrow-e}, \quad G_{\dot{\mathrm{AB}} \mathrm{C} f \dot{\mathrm{D} f}}^{s 4}=2 \Delta^{12} \mathcal{Q}^{13} \mathcal{Q}^{14} .
$$



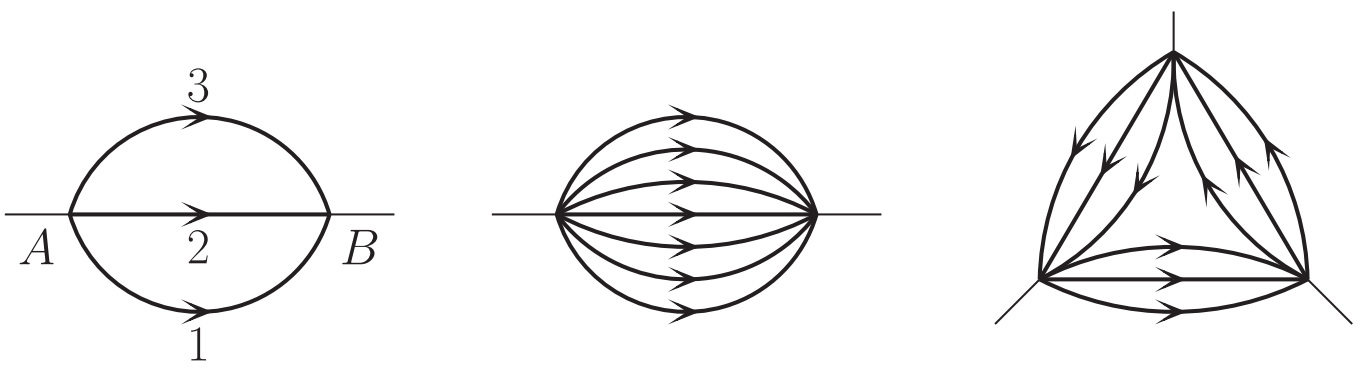

Figure 3. Multiloop diagrams with stacks of internal legs connecting vertices.

With fakeons in 2 and 4 , we have

$$
\begin{aligned}
& G_{\mathrm{ABfCDf}}^{s 4}=-i \mathcal{P}_{4}-\Delta^{13} \mathcal{Q}^{12} \mathcal{Q}^{14}-\Delta^{31} \mathcal{Q}^{32} \mathcal{Q}^{34} \\
& G_{\mathrm{ABfCDf}}^{s 4}=\left.\bar{G}_{\dot{\mathrm{ABfCDf}}}^{s 4}\right|_{e \rightarrow-e}, \quad G_{\text {ÄBfCDf }}^{s 4}=2 \Delta^{13} \mathcal{Q}^{12} \mathcal{Q}^{14} .
\end{aligned}
$$

Finally, with fakeons in three or four legs, we obtain

$$
G_{\mathrm{ABfCfDf}}^{s 4}=G_{\mathrm{AfBfCfDf}}^{s 4}=-i \mathcal{P}_{4}
$$

and no nontrivial cut diagram. This result encodes the purely virtual content of the box diagram.

\section{Simple multiloop diagrams}

In this section we discuss a class of multiloop diagrams that can be treated straightforwardly by generalizing the techniques applied in the previous sections. They are those with stacks of internal legs connecting the same vertices, as shown in figure 3 .

Formula (3.2) shows that, after the integral on the loop energy, the single bubble is equivalent ${ }^{5}$ to a propagator with energy equal to the total incoming energy and frequency equal to the sum of the frequencies. This property iterates to arbitrary stacks of propagators. For example, consider the left two-loop diagram of figure 3, which is the bubble with "diagonal". It leads to the skeleton integral

$$
G_{\mathrm{AB}}^{s 2 D}=\int \frac{\mathrm{d} k^{0}}{2 \pi} \int \frac{\mathrm{d} q^{0}}{2 \pi} \prod_{i=1}^{2} \frac{2 \omega_{i}}{\left(l_{i}-p_{i}\right)^{2}-m_{i}^{2}+i \epsilon_{i}} \frac{2 \omega_{3}}{\left(q+k+p_{3}\right)^{2}-m_{3}^{2}+i \epsilon_{3}},
$$

where $l_{1}=k, l_{2}=q$. Using the residue theorem, we obtain

$$
G_{\mathrm{AB}}^{s 2 D}=\frac{-2 i\left(\omega_{1}+\omega_{2}+\omega_{3}\right)}{\left(e_{1}+e_{2}+e_{3}\right)^{2}-\left(\omega_{1}+\omega_{2}+\omega_{3}\right)^{2}+i\left(\epsilon_{1}+\epsilon_{2}+\epsilon_{3}\right)} .
$$

Again, the stack of propagators is equivalent to a single propagator, from the point of view of the spectral optical theorem, with energy equal to the total incoming energy and frequency equal to the sum of the frequencies, apart from an overall minus sign.

\footnotetext{
${ }^{5}$ Apart from a minus sign, which can be easily handled as an overall factor in the spectral optical theorem.
} 

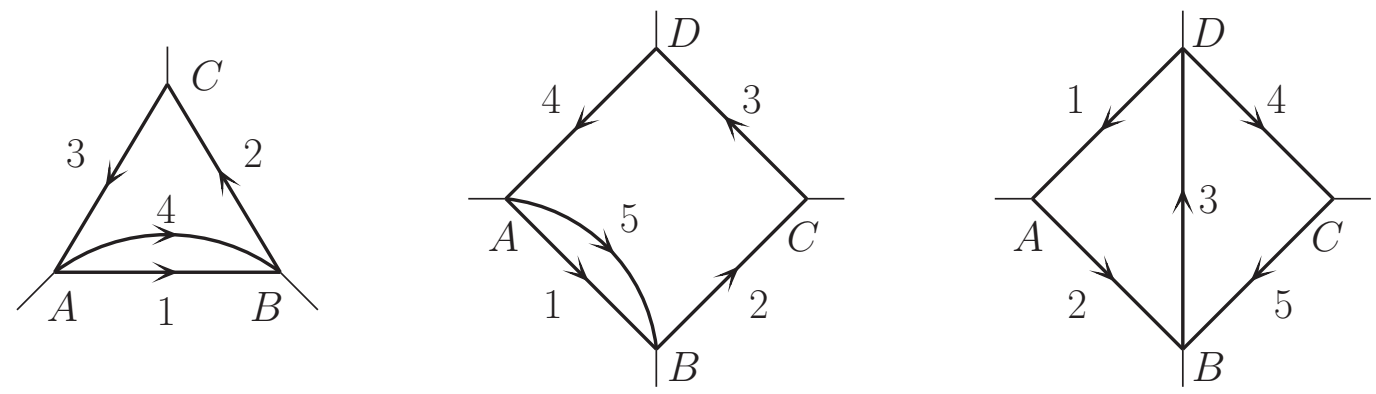

Figure 4. Triangle and box diagrams with "diagonals".

Inside more complicated diagrams, we can replace the stack of propagators with a single propagator, as just shown, and repeat the analyses of the previous sections. For example, the triangle of figure 4 (triangle with "diagonal") is the triangle of section 4 with

$$
e_{1} \rightarrow e_{1}+e_{4}, \quad \omega_{1} \rightarrow \omega_{1}+\omega_{4}
$$

The central diagram of figure 4 is the box of section 5 with

$$
e_{1} \rightarrow e_{1}+e_{5}, \quad \omega_{1} \rightarrow \omega_{1}+\omega_{5} .
$$

It can be used to calculate the left diagram of figure 5, which contributes to the two-loop correction to the self energy.

As far as the fakeon prescription is concerned, it is sufficient to have one fakeon leg in the stack to convert the entire stack into a fakeon. The diagrammatics adapts coherently, since it is impossible to cut a leg belonging to the stack without cutting the whole stack.

\section{Box with diagonal}

A more interesting two-loop diagram is the box with one diagonal, which we denote by $G^{4 D}$. Referring to figure 4, it is identified by the "word" (ABD)(BCD), or, in extended notation, (A2B3D1)(B5C4D3). Below, we just write ABCD. This diagram can be used, for example, to evaluate the right diagram of figure 5 , which is the second contribution to the two-loop self energy. To cover the most general case, we attach external momenta to every vertex.

Choosing the $p_{i}$ orientations opposite to the arrows, the skeleton is

$$
G_{\mathrm{ABCD}}^{s 4 D}=i \int \frac{\mathrm{d} k^{0}}{2 \pi} \int \frac{\mathrm{d} q^{0}}{2 \pi} \frac{2 \omega_{3}}{\left(q+k-p_{3}\right)^{2}-m_{3}^{2}+i \epsilon_{3}} \prod_{i=1,2,4,5} \frac{2 \omega_{i}}{\left(l_{i}-p_{i}\right)^{2}-m_{i}^{2}+i \epsilon_{i}},
$$

where $l_{1}=l_{2}=k, l_{4}=l_{5}=q$. As usual, we first integrate on the loop energies by means of the residue theorem. Then, we use the smart common-denominator identities (2.21) to eliminate the pseudothresholds in favor of the physical thresholds. The result is

$$
G_{\mathrm{ABCD}}^{s 4 D}=-i \sum_{s 4 D} F^{a 3 c}\left[F^{a b} F^{c d}+\frac{1}{2} F^{a b} F^{a 3 d}+\frac{1}{2} F^{b 3 c} F^{c d}+\frac{1}{2} F^{a 3 d} F^{b 3 c}\right],
$$



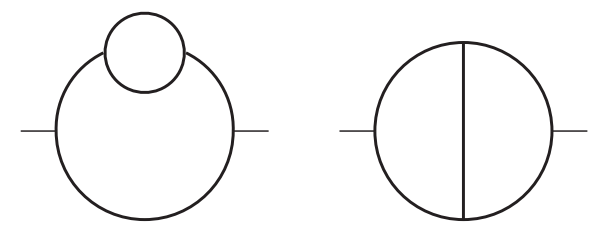

Figure 5. Two-loop corrections to the self energy.

where

$$
F^{a b c}=\frac{1}{e_{a}-e_{b}+e_{c}-\tilde{\omega}_{a}-\tilde{\omega}_{b}-\tilde{\omega}_{c}}
$$

and the sum $\sum_{s 4 D}$ is on $\{a, b\}=p(1,2),\{c, d\}=p(4,5)$, where $p(1,2), p(4,5)$ are the permutations of 1,2 and 4,5 , respectively, plus $(e \rightarrow-e)$.

To write the threshold decomposition, it is convenient to define

$$
\begin{aligned}
Q^{a b c} & =\mathcal{P}\left(\frac{1}{e_{a}-e_{b}+e_{c}-\omega_{a}-\omega_{b}-\omega_{c}}-\frac{1}{e_{a}-e_{b}+e_{c}-\omega_{a}+\omega_{b}-\omega_{c}}\right), \\
\Delta^{a b c} & =\pi \delta\left(e_{a}-e_{b}+e_{c}-\omega_{a}-\omega_{b}-\omega_{c}\right), \quad \hat{\Delta}^{a b c}=\pi \delta\left(e_{a}-e_{b}+e_{c}+\omega_{a}+\omega_{b}+\omega_{c}\right) .
\end{aligned}
$$

As usual, the threshold decomposition is worked out by using (2.15) and writing the various contributions in manifest diagrammatic forms by means of identities like (2.19). The result is

$$
\begin{aligned}
G_{\mathrm{ABCD}}^{s 4 D}= & -i \mathcal{P}_{\mathrm{ABCD}}^{s 4 D}-\left.\Delta^{12} \mathcal{P}_{345}\right|_{e_{3} \rightarrow e_{3}-e_{2}-\omega_{2}}-\left.\Delta^{45} \mathcal{P}_{123}\right|_{e_{3} \rightarrow e_{3}-e_{5}-\omega_{5}}-\left.\Delta^{21} \mathcal{P}_{345}\right|_{e_{3} \rightarrow e_{3}-e_{1}-\omega_{1}} \\
& -\left.\Delta^{54} \mathcal{P}_{123}\right|_{e_{3} \rightarrow e_{3}-e_{4}-\omega_{4}}-\sum_{s 4 D} \Delta^{a 3 c} \mathcal{Q}^{a b} \mathcal{Q}^{c d}+i \sum_{s 4 D} \Delta^{a 3 c}\left(\Delta^{a b} \mathcal{Q}^{c d}+\mathcal{Q}^{a b} \Delta^{c d}\right) \\
& +\frac{i}{2} \sum_{s 4 D}\left[\mathcal{Q}^{a 3 c} \Delta^{a b} \Delta^{c d}+\Delta^{a 3 c}\left(\mathcal{Q}^{a b} \Delta^{a 3 d}+\Delta^{b 3 c} \mathcal{Q}^{c d}\right)\right] \\
& +\sum_{s 4 D} \Delta^{a 3 c}\left[\Delta^{a b} \Delta^{c d}+\frac{1}{2} \Delta^{a b} \Delta^{a 3 d}+\frac{1}{2} \Delta^{b 3 c} \Delta^{c d}\right]
\end{aligned}
$$

where $\mathcal{P}_{\mathrm{ABCD}}^{s 4 D}=\left.i G_{\mathrm{ABCD}}^{s 4 D}\right|_{F \rightarrow \mathcal{P}}$ and $-i \mathcal{P}_{123},-i \mathcal{P}_{345}$ are the purely virtual contents of the triangle diagrams with legs $1,2,3$ and $3,4,5$, respectively (see section 4 , formula (4.4)). The decompositions of the cut diagrams are

$$
\begin{aligned}
& G_{\dot{\mathrm{ABCD}}}^{s 4 D}=\left.2 i \Delta^{21} T_{345}^{s}\right|_{e_{3} \rightarrow e_{3}-e_{1}-\omega_{1}}, \quad G_{\mathrm{A \dot {B } \dot { D }}}^{s 4 D}=\left.\bar{G}_{\dot{\mathrm{ABCD}}}^{s 4 D}\right|_{e \rightarrow-e}, \quad G_{\mathrm{ABC \dot { }}}^{s 4 D}=\left.G_{\dot{\mathrm{ABCD}}}^{s 4 D}\right|_{2 \leftrightarrow 5} ^{1 \leftrightarrow 4}, \\
& G_{\dot{\mathrm{A} \dot{B} C \dot{D}}}^{s 4 D}=\left.G_{\mathrm{A} \dot{\mathrm{B}} \dot{\mathrm{D}}}^{s 4 D}\right|_{2 \leftrightarrow 5} ^{1 \leftrightarrow 4}, \quad G_{\mathrm{ABCD}}^{s 4 D}=\left.\bar{G}_{\dot{\mathrm{AB}} \mathrm{C} \dot{\mathrm{C}}}^{s 4 D}\right|_{4 \leftrightarrow 5} ^{1 \leftrightarrow 2}, \quad G_{\dot{\mathrm{A} \dot{B} \dot{C} \mathrm{D}}}^{s 4 D}=\left.\bar{G}_{\mathrm{A} \dot{\mathrm{B} C D}}^{s 4 D}\right|_{4 \leftrightarrow 5} ^{1 \leftrightarrow 2}, \\
& G_{\mathrm{ABCD}}^{s 4 D}=2 \hat{\Delta}^{235}\left(\hat{\mathcal{Q}}^{21}-i \Delta^{12}-i \hat{\Delta}^{135}\right)\left(\hat{\mathcal{Q}}^{54}-i \Delta^{45}-i \hat{\Delta}^{234}\right), \quad G_{\dot{\mathrm{A} B \dot{C}} \dot{D}}^{s 4 D}=\left.\bar{G}_{\mathrm{ABCD}}^{s 4 D}\right|_{e \rightarrow-e}, \\
& G_{\dot{\mathrm{A} \dot{\mathrm{B} C D}}}^{s 4 D}=2 \hat{\Delta}^{135}\left(\hat{\mathcal{Q}}^{12}+i \Delta^{21}+i \hat{\Delta}^{235}\right)\left(\hat{\mathcal{Q}}^{54}-i \Delta^{45}-i \hat{\Delta}^{134}\right), \quad G_{\mathrm{ABC} \dot{D}}^{s 4 D}=\left.\bar{G}_{\dot{\mathrm{A} \dot{\mathrm{B} C D}}}^{s 4 D}\right|_{e \rightarrow-e}, \\
& G_{\mathrm{AB \dot {C }}}^{s 4 D}=2 \hat{\Delta}^{234}\left(\hat{\mathcal{Q}}^{21}-i \Delta^{12}-i \hat{\Delta}^{134}\right)\left(\hat{\mathcal{Q}}^{45}+i \Delta^{54}+i \hat{\Delta}^{235}\right), \quad G_{\dot{\mathrm{A} B C D}}^{s 4 D}=\left.\bar{G}_{\mathrm{A \dot {B }} \dot{\mathrm{C} D}}^{s 4 D}\right|_{e \rightarrow-e}, \\
& G_{\dot{\mathrm{ABC}}}^{s 4 D}=4 i \Delta^{21} \Delta^{54}\left(\mathcal{Q}^{235}-i \Delta^{235}-i \hat{\Delta}^{134}\right), \quad G_{\mathrm{ABCD}}^{s 4 D}=\left.\bar{G}_{\dot{\mathrm{AB}} \mathrm{C} D}^{s 4 D}\right|_{e \rightarrow-e},
\end{aligned}
$$


where $T_{345}^{s}$ is the triangle (4.4) with legs $3,4,5$. The conjugate uncut diagram is $G_{\dot{\mathrm{A}} \dot{\mathrm{B}} \dot{\mathrm{C}} \dot{\mathrm{D}}}^{s 4 D}=$ $\bar{G}_{\mathrm{ABCD}}^{s 4 D}$.

The spectral optical theorem reads

$$
\begin{aligned}
& G_{\mathrm{ABCD}}^{s 4 D}+G_{\dot{\mathrm{A} \dot{\mathrm{B}} \dot{ }} \dot{D}}^{s 4 D}+G_{\dot{\mathrm{A}} \mathrm{BCD}}^{s}+G_{\mathrm{A} \dot{\mathrm{B}} \dot{\mathrm{C}}}^{s 4 D}+G_{\mathrm{ABCD}}^{s 4 D}+G_{\dot{\mathrm{A} \dot{\mathrm{B} C D}}}^{s 4 D}+G_{\mathrm{ABCD}}^{s 4 D}+G_{\dot{\mathrm{A} \dot{\mathrm{B}} \mathrm{C}}}^{s 4 D}+G_{\mathrm{A} \dot{\mathrm{B} C D}}^{s 4 D} \\
& +G_{\dot{\mathrm{A}} \dot{\mathrm{C} \dot{\mathrm{D}}}}^{s 4 D}+G_{\dot{\mathrm{A} \dot{\mathrm{B} C D}}}^{s 4 D}+G_{\mathrm{AB} \dot{\mathrm{C} \dot{D}}}^{s 4 D}+G_{\mathrm{A} \dot{\mathrm{B}} \dot{\mathrm{C} D}}^{s 4 D}+G_{\dot{\mathrm{ABCD}}}^{s 4 D}+G_{\dot{\mathrm{ABCD}}}^{s 4 D}+G_{\mathrm{ABCD}}^{s 4 D}=0
\end{aligned}
$$

and can be easily verified. The spectral optical identities are the various threshold contributions to this equality, which vanish separately.

\subsection{Fakeons}

The fakeon projections can now be implemented straightforwardly. If leg 1, which is the segment DA, is a fakeon, we suppress all the contributions proportional to $\Delta^{a b}, \Delta^{a b c}$ and $\hat{\Delta}^{a b c}$, whenever $a, b$ or $c$ are equal to 1 . Denoting this case by fABCD, the uncut diagram is

$$
\begin{aligned}
G_{\mathrm{fABCD}}^{s 4 D}= & -i \mathcal{P}_{\mathrm{ABCD}}^{s 4 D}-\Delta^{45}\left(\left.\mathcal{P}_{123}\right|_{e_{3} \rightarrow e_{3}-e_{5}-\omega_{5}}-i \Delta^{234} \mathcal{Q}^{21}\right)-\Delta^{234} \mathcal{Q}^{21}\left(\mathcal{Q}^{45}-i \Delta^{235}\right) \\
& -\Delta^{54}\left(\left.\mathcal{P}_{123}\right|_{e_{3} \rightarrow e_{3}-e_{4}-\omega_{4}}-i \hat{\Delta}^{234} \hat{\mathcal{Q}}^{21}\right)-\hat{\Delta}^{234} \hat{\mathcal{Q}}^{21}\left(\hat{\mathcal{Q}}^{45}-i \hat{\Delta}^{235}\right) \\
& -\Delta^{235} \mathcal{Q}^{21}\left(\mathcal{Q}^{54}-i \Delta^{54}\right)-\hat{\Delta}^{235} \hat{\mathcal{Q}}^{21}\left(\hat{\mathcal{Q}}^{54}-i \Delta^{45}\right) .
\end{aligned}
$$

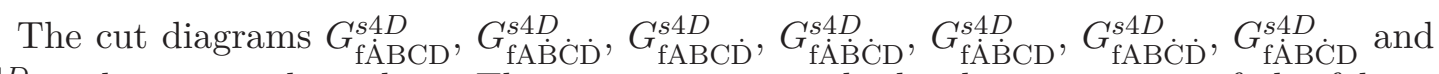
$G_{\mathrm{fABCD}}^{s 4 D}$ disappear altogether. This is consistent with the diagrammatics of the fakeon prescription, since those diagrams contain a cut fakeon leg and by (2.13) the cut fakeon propagator vanishes. The surviving cut diagrams are

$$
\begin{aligned}
& G_{\mathrm{fABC} D}^{s 4 D}=2 \Delta^{54}\left(\left.\mathcal{P}_{123}\right|_{e_{3} \rightarrow e_{3}-e_{4}-\omega_{4}}-i \Delta^{235} \mathcal{Q}^{21}-i \hat{\Delta}^{234} \hat{\mathcal{Q}}^{21}\right), \\
& G_{\mathrm{f \dot {A }} \dot{\mathrm{B} C \dot{D}}}^{s 4 D}=2 \Delta^{45}\left(\left.\mathcal{P}_{123}\right|_{e_{3} \rightarrow e_{3}-e_{5}-\omega_{5}}+i \Delta^{234} \mathcal{Q}^{21}+i \hat{\Delta}^{235} \hat{\mathcal{Q}}^{21}\right), \\
& G_{\mathrm{fA} \dot{\mathrm{B} C D}}^{s 4 D}=2 \hat{\Delta}^{235} \hat{\mathcal{Q}}^{21}\left(\hat{\mathcal{Q}}^{54}-i \Delta^{45}-i \hat{\Delta}^{234}\right), \quad G_{\mathrm{fAB} \dot{\mathrm{C} \dot{D}}}^{s 4 D}=2 \Delta^{235} \mathcal{Q}^{21}\left(\mathcal{Q}^{54}+i \Delta^{54}+i \Delta^{234}\right), \\
& G_{\mathrm{fA} \dot{\mathrm{B}} \dot{\mathrm{C} D}}^{s 4 D}=2 \hat{\Delta}^{234} \hat{\mathcal{Q}}^{21}\left(\hat{\mathcal{Q}}^{45}+i \Delta^{54}+i \hat{\Delta}^{235}\right), \quad G_{\mathrm{fA்BC}}^{s 4 D}=2 \Delta^{234} \mathcal{Q}^{21}\left(\mathcal{Q}^{45}-i \Delta^{45}-i \Delta^{235}\right),
\end{aligned}
$$

It is easy to show that these formulas also follow from the diagrammatic rules given in subsection 2.2. In particular, the fakeon propagator of every link agrees with (2.14).

The spectral optical theorem reads

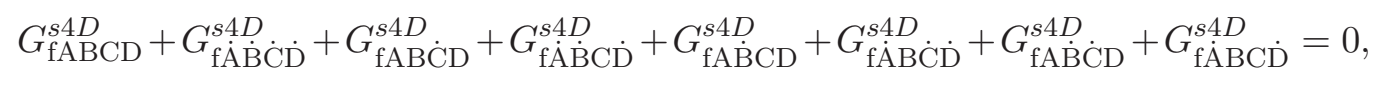

where $G_{\mathrm{fA்} \dot{\mathrm{B}} \dot{\mathrm{D}}}^{s 4 D}=\bar{G}_{\mathrm{fABCD}}^{s 4 D}$, and can be verified straightforwardly.

If leg 3 is a fakeon, we denote the diagram by $\mathrm{AB}|\mathrm{f}| \mathrm{CD}$. We have

$$
\begin{aligned}
G_{\mathrm{AB}|\mathrm{f}| \mathrm{CD}}^{s 4 D}= & -i \mathcal{P}_{\mathrm{ABCD}}^{s 4 D}-\left.\Delta^{12} \mathcal{P}_{345}\right|_{e_{3} \rightarrow e_{3}-e_{2}-\omega_{2}}-\left.\Delta^{45} \mathcal{P}_{123}\right|_{e_{3} \rightarrow e_{3}-e_{5}-\omega_{5}} \\
& -\left.\Delta^{21} \mathcal{P}_{345}\right|_{e_{3} \rightarrow e_{3}-e_{1}-\omega_{1}}-\left.\Delta^{54} \mathcal{P}_{123}\right|_{e_{3} \rightarrow e_{3}-e_{4}-\omega_{4}}+\frac{i}{2} \sum_{s 4 D} \mathcal{Q}^{a 3 c} \Delta^{a b} \Delta^{c d}
\end{aligned}
$$


and $G_{\dot{\mathrm{A}} \dot{\mathrm{B}}|\mathrm{f}| \dot{\mathrm{C}} \dot{\mathrm{D}}}^{s 4 D}=\bar{G}_{\mathrm{AB}|\mathrm{f}| \mathrm{CD}}^{s 4 D}$. The nonvanishing cut diagrams are

$$
\begin{aligned}
& G_{\dot{\mathrm{AB}}|\mathrm{f}| \mathrm{CD}}^{s}=2 \Delta^{21}\left(\left.\mathcal{P}_{345}\right|_{e_{3} \rightarrow e_{3}-e_{1}-\omega_{1}}-i \Delta^{45} \mathcal{Q}^{234}-i \Delta^{54} \mathcal{Q}^{235}\right),
\end{aligned}
$$

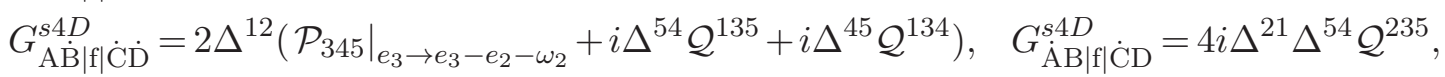

$$
\begin{aligned}
& G_{\mathrm{AB}|\mathrm{f}| \dot{\mathrm{C} D}}^{s}=2 \Delta^{54}\left(\left.\mathcal{P}_{123}\right|_{e_{3} \rightarrow e_{3}-e_{4}-\omega_{4}}-i \Delta^{12} \mathcal{Q}^{135}-i \Delta^{21} \mathcal{Q}^{235}\right), \quad G_{\mathrm{A} \dot{\mathrm{B}}|\mathrm{C}| \mathrm{C} \dot{\mathrm{D}}}^{s+D}=-4 i \Delta^{12} \Delta^{45} \mathcal{Q}^{134}, \\
& G_{\dot{\mathrm{A}} \mathrm{B}|\mathrm{f}| \mathrm{CD}}^{s+\dot{D}}=2 \Delta^{45}\left(\left.\mathcal{P}_{123}\right|_{e_{3} \rightarrow e_{3}-e_{5}-\omega_{5}}+i \Delta^{21} \mathcal{Q}^{234}+i \Delta^{12} \mathcal{Q}^{134}\right),
\end{aligned}
$$

which agree with the diagrammatic rules of the fakeon prescription.

The spectral optical theorem reads

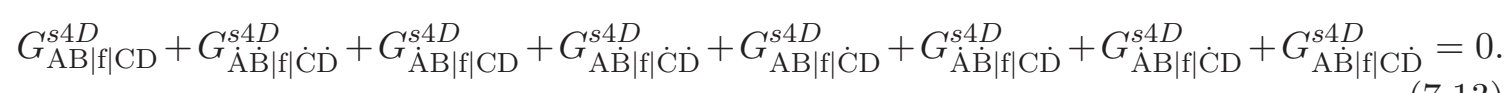

The other possibilities of distributing fakeons in the internal legs can be treated similarly. The case where all the internal legs are fakeons gives the purely virtual content of the box with diagonal, which is

$$
\left.G_{\mathrm{ABCD}}^{s 4 D}\right|_{\text {purely virtual }}=-i \mathcal{P}_{\mathrm{ABCD}}^{s 4 D}
$$

\section{Further insight into the algebraic structure of the spectral optical iden- tities}

In this section we provide more insight into the algebraic structure of the spectral optical identities and work out formulas for more complicated diagrams, like the pentagon and the hexagon.

It is useful to introduce a few definitions, such as

$$
\begin{aligned}
I_{N} & =\sum_{p_{N}} \prod_{k=2}^{N} F^{a_{1} a_{k}}+(e \rightarrow-e), \quad J_{N}=\sum_{p_{N}} F^{a_{N} a_{N-1}} \prod_{k=2}^{N-1} F^{a_{1} a_{k}}+(e \rightarrow-e), \\
K_{N} & =\sum_{p_{N}} F^{a_{1} a_{2}} F^{a_{3} a_{2}} F^{a_{3} a_{4}} F^{a_{5} a_{4}} \cdots+(e \rightarrow-e),
\end{aligned}
$$

where $p_{N}$ denotes the permutations of $\left\{a_{1} \cdots a_{N}\right\}$.

The triangle, box, pentagon and hexagon diagrams of figure 6 give

$$
\begin{aligned}
G_{3}^{s} & =-\frac{i}{2 !} I_{3}, \quad G_{4}^{s}=-\frac{i}{3 !}\left(I_{4}+\frac{3}{2} J_{4}\right), \quad G_{5}^{s}=-\frac{i}{5 !}\left(I_{5}+4 J_{5}+2 K_{5}\right), \\
G_{6}^{s} & =-\frac{i}{6 !}\left(I_{6}+5 J_{6}+5 X_{6}+10 Y_{6}\right),
\end{aligned}
$$

where

$$
\begin{aligned}
X_{6}=\sum_{p_{N}} F^{a b} F^{a c} F^{a d} F^{e d} F^{e f}+(e \rightarrow-e), & Y_{6}=\sum_{p_{N}} F^{a b} F^{a c} F^{a d} F^{e d} F^{f c}+(e \rightarrow-e), \\
Z_{6}=\sum_{p_{N}} F^{a b} F^{a c} F^{a d} F^{e d} F^{f d}+(e \rightarrow-e), & Z_{6}+K_{6}=2 Y_{6} .
\end{aligned}
$$



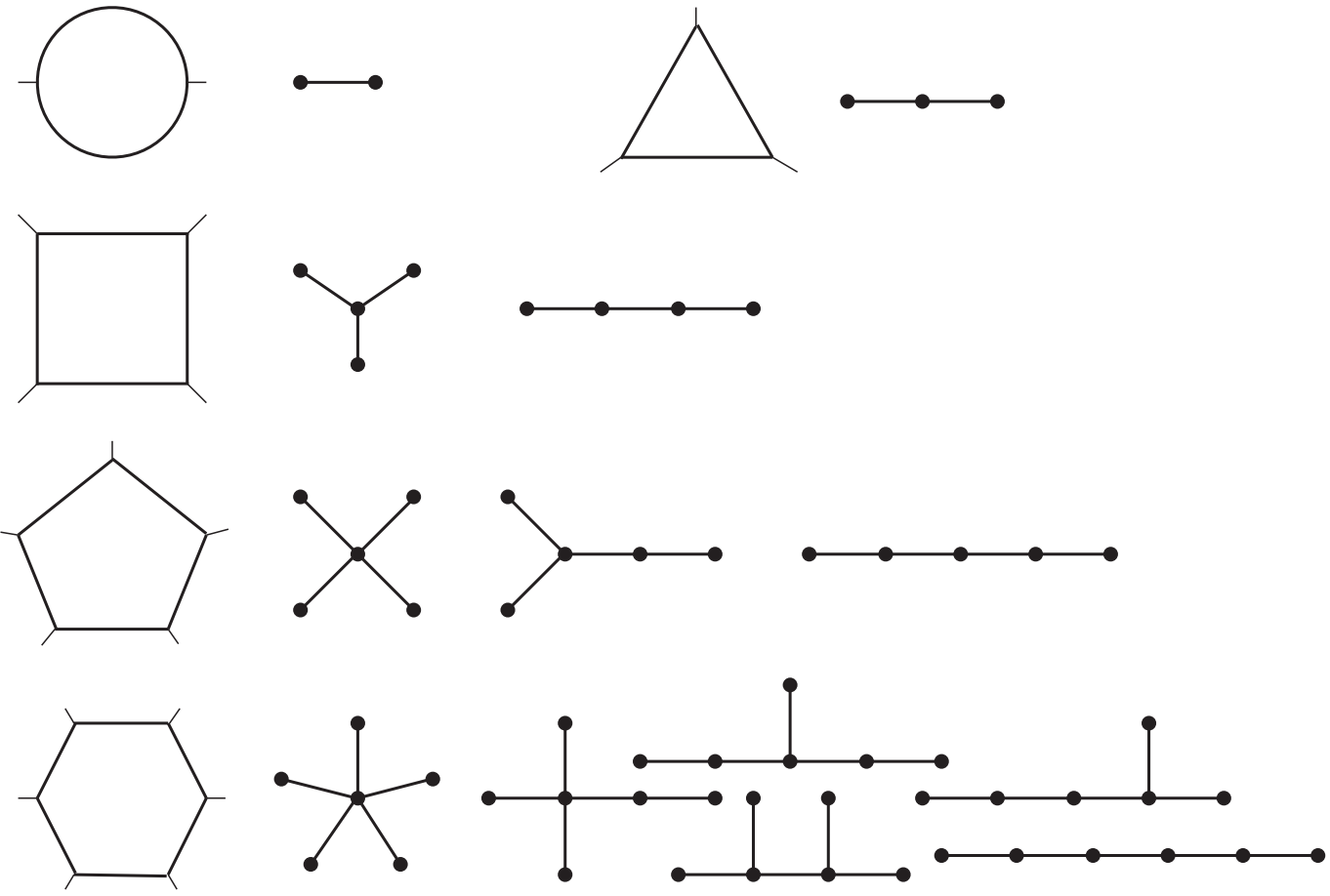

Figure 6. One-loop diagrams and their snowflake versions.

The various contributions can be represented by means of the "snowflake diagrams" shown in figure 6 , where each leg is an $F^{a b}$ and the vertices are repeated indices. Oriented legs can be used to distinguish $F^{a b}$ from $F^{b a}$.

In general, the multithresholds can be expressed in more than one equivalent ways. This fact leads to nontrivial identities, such as the last of (8.3) and

$$
F^{12} F^{13} F^{43}+F^{12} F^{42} F^{43}-F^{12} F^{13} F^{42}-F^{13} F^{42} F^{43}=0 .
$$

Now we show that the threshold decomposition and the fakeon projection are not affected by this.

Step 4.b) of subsection 2.5 tells us how to arrange the levels $\ell \geqslant 1$ of the decomposition. The proper diagrammatic structure of the $\ell=0$ sector, instead, is defined by the procedure itself. The threshold decomposition of (8.4) gives the second identity of (2.19) at level 0 , that is to say,

$$
\mathcal{P}^{12} \mathcal{P}^{13} \mathcal{P}^{43}+\mathcal{P}^{12} \mathcal{P}^{42} \mathcal{P}^{43}-\mathcal{P}^{12} \mathcal{P}^{13} \mathcal{P}^{42}-\mathcal{P}^{13} \mathcal{P}^{42} \mathcal{P}^{43}=0
$$

If we add the left-hand side to the purely virtual part of the box diagram, we change its form, but not its value, because the right-hand side of (8.5) vanishes identically. For the same reason, no remnants drop to the higher levels. Similar arguments apply to the identity of (8.3) for the hexagon diagram.

A source of worry could come from the other identities of (2.19), where the right-hand side is nonvanishing and different levels can mix. Those identities, however, require an odd 
number of internal legs (triangle, pentagon, etc.), i.e., products of an even number of $F^{a b}$. This makes relations like (8.4) unavailable: it is impossible to arrange the product of an even number of $F^{a b}$ without involving pseudothresholds, which have disappeared after step 3 ) of subsection 2.5. In the end, the threshold decomposition is unambiguous.

We conclude by discussing the algebraic structure of the cut diagrams. In the case of the triangle, the key identities are

$$
\alpha \beta-\bar{\alpha} \bar{\beta}=(\alpha-\bar{\alpha}) \beta+\bar{\alpha}(\beta-\bar{\beta})=\alpha(\beta-\bar{\beta})+(\alpha-\bar{\alpha}) \bar{\beta}
$$

where $\alpha$ and $\beta$ are arbitrary complex numbers and $\bar{\alpha}, \bar{\beta}$ are their complex conjugates. Choosing, e.g., $\alpha=F^{12}$ and $\beta=F^{13}$ and multiplying by a further factor $-i$, we get, from the first equality,

$$
-i F^{12} F^{13}+i \bar{F}^{12} \bar{F}^{13}=-2 \Delta^{12} F^{13}-2 \bar{F}^{12} \Delta^{13} .
$$

The differences $\alpha-\bar{\alpha}$ and $\beta-\bar{\beta}$ give the $\delta$ functions belonging to the cut propagators. The application of identities like these to (4.3) gives a different procedure to obtain the spectral optical identities of the triangle, collected in table 2 .

In the case of the box, we can manipulate (5.1) by means of identities like

$$
\begin{aligned}
\alpha \beta \gamma-\bar{\alpha} \bar{\beta} \bar{\gamma} & =(\alpha-\bar{\alpha}) \beta \gamma+\bar{\alpha}(\beta-\bar{\beta}) \gamma+\bar{\alpha} \bar{\beta}(\gamma-\bar{\gamma}) \\
& =\alpha \beta(\gamma-\bar{\gamma})+\alpha(\beta-\bar{\beta}) \bar{\gamma}+(\alpha-\bar{\alpha}) \bar{\beta} \bar{\gamma} \\
& =\alpha(\beta-\bar{\beta}) \gamma+(\alpha-\bar{\alpha}) \bar{\beta} \gamma+\bar{\alpha} \bar{\beta}(\gamma-\bar{\gamma}) \\
& =(\alpha-\bar{\alpha}) \bar{\beta} \gamma+\alpha(\beta-\bar{\beta}) \bar{\gamma}+\bar{\alpha} \beta(\gamma-\bar{\gamma})+(\alpha-\bar{\alpha})(\beta-\bar{\beta})(\gamma-\bar{\gamma})
\end{aligned}
$$

and then apply the threshold decomposition. Note that the last identity involves cut diagrams with two marked and two unmarked vertices (such as $G_{\dot{\mathrm{ABCD}}}^{s 4}$ ).

We can proceed similarly for the pentagon and the hexagon, as well as for the multiloop diagrams. Identities like (8.6) and (8.8) can be used to provide alternative proofs of the optical theorem (via its spectral version and the spectral optical identities).

In the end, the algebraic structure of the spectral optical identities is encoded in simple relations such as (8.6) and (8.8). The counterpart of this simplicity is a lengthier diagrammatics, in the sense that each ordinary diagram is expanded into the sum of numerous "snowflake" diagrams, as shown in figure 6 .

\section{Generalization to propagators with arbitrary real residues}

In this section we generalize the spectral optical identities to propagators with arbitrary real residues at the poles. This allows us to treat particles and antiparticles asymmetrically and shows that the identities do not rely on Lorentz invariance, nor the CPT theorem. 
Define the propagators

$$
\begin{aligned}
& \underline{p \rightarrow}=\frac{i \xi}{e-\omega+i \epsilon}-\frac{i \zeta}{e+\omega-i \epsilon}, \\
& \stackrel{p \rightarrow}{\bullet}=\frac{i \zeta}{e+\omega+i \epsilon}-\frac{i \xi}{e-\omega-i \epsilon},
\end{aligned}
$$

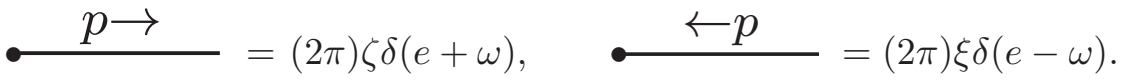

instead of (2.12), where $\xi$ and $\zeta$ are arbitrary real numbers.

For the threshold decompositions, it is convenient to introduce the quantities

$$
\begin{aligned}
F^{a b} & =\frac{\xi_{b}}{e_{a}-e_{b}-\tilde{\omega}_{a}-\tilde{\omega}_{b}}, & \mathcal{P}^{a b} & =\mathcal{P} \frac{\xi_{b}}{e_{a}-e_{b}-\omega_{a}-\omega_{b}}, \\
\mathcal{Q}^{a b} & =\mathcal{P}^{a b}-\mathcal{P} \frac{\zeta_{b}}{e_{a}-e_{b}-\omega_{a}+\omega_{b}}, & \Delta^{a b} & =\pi \zeta_{a} \xi_{b} \delta\left(e_{a}-e_{b}-\omega_{a}-\omega_{b}\right),
\end{aligned}
$$

instead of (2.20). In the formulas of this section it is understood that every expression must be expanded and, after the expansion, every power of $\xi_{a}$ with the same index $a$ must be turned into $\xi_{a}$. The same must be done for the powers of $\zeta_{a}$. For example, $\Delta^{a b} \Delta^{a c}$ is a double $\delta$ function multiplied by $\pi^{2} \zeta_{a} \xi_{b} \xi_{c}$, instead of $\pi^{2} \zeta_{a}^{2} \xi_{b} \xi_{c}$. With these conventions, it is easy to show that formula (5.1) for the box skeleton turns into

$$
G_{\mathrm{ABCD}}^{s 4}=-\frac{i}{6} \sum_{\text {perms }} \zeta_{a} F^{a b} F^{a c} F^{a d}-\frac{i}{4} \sum_{\text {perms }} \zeta_{a} \zeta_{d} F^{a b} F^{a c} F^{d b}+(e \rightarrow-e, \xi \leftrightarrow \zeta) .
$$

Moreover, the threshold decompositions (5.9) and (5.11) remain formally the same, like all the other formulas of section 5 , including those referring to the fakeon prescription.

The example of the box illustrates how to proceed for every diagram, when we want to treat propagators with arbitrary real residues.

\section{Diagrams with nontrivial numerators and degenerate diagrams}

So far, we have considered diagrams generated by vertices that do not carry derivatives. Derivative vertices bring nontrivial numerators into the integrands. The Passarino-Veltman reduction allows us to convert one-loop diagrams with arbitrary numerators into linear combinations of diagrams with unit numerators [36]. However, it does not work for arbitrary diagrams. In particular, it may fail with more loops.

The versatility of the spectral optical identities provides a more powerful way out. Recall that every identity holds without integrating on the space components $\mathbf{k}_{l}$ of the loop momenta. This means that the functions of $\mathbf{k}_{l}$ can be factored out. A nontrivial numerator in a loop integral is a tensorial polynomial $N(p, k)$ of the external momenta $p$ and the loop momenta $k$. Let us expand it as a sum of monomials

$$
\prod_{j} p_{j}^{\mu_{j}} \prod_{l} k_{l}^{\mu_{l}}
$$

Since we can factor out the components of $p$ and every space components of $k$, we just need to pay attention to the loop energies $k_{l}^{0}$. They can be used to simplify the poles of 
the propagators (2.12) and reduce the integral to a sum of integrals with fewer internal legs and/or fewer loop energies in the numerators. ${ }^{6}$

Inside the integrals obtained this way, we may not find the propagators (2.12) and (2.14), since the poles at $e= \pm \omega$ need not appear in those combinations. Yet, we can use the generalization of the previous section, since formulas (9.1) with appropriate choices of $\xi$ or $\zeta$ suit every case.

Iterating this procedure, we reduce to a linear combination of spectral optical identities of the types already considered. This proves that they hold for derivative vertices and diagrams with arbitrary numerators.

Another important point is that the identities derived so far have are valid under a tacit "non-coincidence" assumption, which means that the thresholds are all distinct. The simplest way to fulfill this requirement is to imagine that the propagators have nonvanishing, different masses $m_{i}$, such that the sums $\sum_{i \in J} m_{i}$ are all different, for every subset $J$ of internal legs. ${ }^{7}$ Another possibility is to assume, as we have done so far, that each vertex carries an external leg, equipped with an independent external momentum. For example, instead of the double bubble $10 \alpha$, where the central vertex is attached to internal legs only, we take $\backslash O \propto$, with additional external legs stemming from the central vertex.

Yet, in many physical situations identical propagators appear, as in the left diagram of figure 5, and identical thresholds. The square of a propagator defined by the Feynman prescription is well defined. What about the threshold decomposition and the fakeon prescription? The square of the Cauchy principal value is ill-defined and so is the square of a Dirac $\delta$ function.

The way out is to use a "coincidence splitting method" (which works for powers of the Feynman propagator as well). In other words, we view coinciding thresholds as the limits of distinct thresholds, obtained in the ways described above (i.e., by inserting fictitious, small mass differences or equipping the vertices with additional external legs flowing in small momenta). For example, we can view the left diagram of figure 5 as a limit of the middle diagram of figure 4 .

Then, arbitrary powers of the Cauchy principal value are well defined, since [40]

$$
\lim _{\epsilon \rightarrow 0} \mathcal{P} \prod_{i=1}^{n+1} \frac{1}{x-\epsilon c_{i}}=\frac{(-1)^{n}}{n !} \frac{\mathrm{d}^{n}}{\mathrm{~d} x^{n}} \mathcal{P} \frac{1}{x}
$$

where $c_{i}$ are distinct numbers. The coincidence splitting method trivializes the powers of a $\delta$ function:

$$
\lim _{\epsilon \rightarrow 0} \prod_{i=1}^{n+1} \delta\left(x-\epsilon c_{i}\right)=0 .
$$

\footnotetext{
${ }^{6}$ If the power of some loop energy $k^{0}$ is large enough, remnants with no $k$-dependent denominators can survive. These contributions can be dropped. If the surviving power of $k^{0}$ is odd, they vanish by symmetric integration. If the surviving power of $k^{0}$ is even, they are killed by the dimensional regularization, since they give integrals of polynomials of $k$ in $\mathrm{d}^{D} k /(2 \pi)^{D}$.

${ }^{7}$ To prove the optical theorem in the presence of massless fields, it is convenient to equip them with small, fictitious masses, otherwise the asymptotic states of other particles (e.g., the electron in QED) are ill defined. The non coincidence assumption can be fulfilled using the fictitious masses. The massless limit is studied after taking care of the infrared divergences (see section 12).
} 
The Feynman prescription generates products of delta functions times principal values, but only in well-defined combinations such as

$$
\lim _{\epsilon \rightarrow 0}\left(\frac{\delta\left(x-\epsilon c_{1}\right)}{x-\epsilon c_{2}}+\frac{\delta\left(x-\epsilon c_{2}\right)}{x-\epsilon c_{1}}\right)=\lim _{\epsilon \rightarrow 0} \frac{\delta\left(x-\epsilon c_{1}\right)-\delta\left(x-\epsilon c_{2}\right)}{\epsilon\left(c_{1}-c_{2}\right)}=-\delta^{\prime}(x) .
$$

The fakeon prescription leaves or drops both contributions appearing on the left-hand side, so it is also well defined.

\section{Thick fakeons}

In this section we generalize the identities to complex fakeon frequencies and study the thick fakeons, which have nonvanishing widths at the tree level. An example is offered by a propagator of the form

$$
\frac{2 i M^{2}}{\left(p^{2}-\mu^{2}\right)^{2}+M^{4}}=\frac{1}{p^{2}-\mu^{2}-i M^{2}}-\frac{1}{p^{2}-\mu^{2}+i M^{2}},
$$

where, as usual, $p^{\mu}=(e, \mathbf{p})$. We can view it as the difference $P_{\text {thick }}-\bar{P}_{\text {thick }}$, where

$$
P_{\text {thick }}=\frac{i}{2 \Omega}\left(\frac{i}{e-\Omega}-\frac{i}{e+\Omega}\right)
$$

and $\Omega=\sqrt{\mathbf{p}^{2}+\mu^{2}-i M^{2}}$. The propagator $P_{\text {thick }}$ is of the type already studied, apart from two features: the complex frequency $\Omega$ and the overall factor $i /(2 \Omega)$.

More generally, the typical propagator of thick fakeons reads

$$
\frac{C}{p^{2}-\mu^{2}-i M^{2}}-\frac{C^{*}}{p^{2}-\mu^{2}+i M^{2}}=C^{*} P_{\text {thick }}-C \bar{P}_{\text {thick }},
$$

where $C$ is a complex number. Another interesting example is

$$
\frac{i\left(p^{2}-\mu^{2}\right)}{\left(p^{2}-\mu^{2}\right)^{2}+M^{4}}=\frac{i}{2}\left(\frac{1}{p^{2}-\mu^{2}-i M^{2}}+\frac{1}{p^{2}-\mu^{2}+i M^{2}}\right)=-\frac{i}{2}\left(P_{\text {thick }}+\bar{P}_{\text {thick }}\right) .
$$

Although (11.3) and (11.4) cannot be obtained from a local Lagrangian, they can be used to include certain limitations due to the experimental apparatus, like the energy resolution around the "fakeon peak".

We show that the spectral optical identities for diagrams involving propagators like (11.1), (11.3) and (11.4) can be derived as (complex) linear combinations of (the complexified versions of) the identities derived in the previous sections.

Typically, propagators like (11.1) appear in higher-derivative theories. Examples are the Lee-Wick (LW) models [10, 12-15]. It is worth to emphasize, though, that the LW models do not have fakeons, but unstable ghosts, which are not really out of the physical spectrum (in the same way as the muon is not out of the physical spectrum of the standard model). The LW ghosts can be dropped from the spectrum only in an effective field theory approach, if they have relatively large decay widths.

Instead, fakeons are eradicated from the theory at the fundamental level (and therefore, at all energies). Due to this, they do not need to decay. For example, in the models 
of [24], which have a $\mathbb{Z}_{2}$ symmetric fakeon sector, they have indentically vanishing widths. Moreover, fakeons do not need higher derivatives, nor negative residues in front of their propagators. What makes all this possible is the fakeon projection, which is consistent only if we adopt the fakeon quantization prescription. ${ }^{8}$

As shown by Piva and the current author in $[16,17]$, it is possible to reformulate the Lee-Wick models, and a variety of other higher-derivative theories, by converting them into theories of particles and thick fakeons. This is one reason why it is interesting to generalize the results of the previous sections to this type of fakeons. Other reasons will be mentioned soon.

The generalization proceeds as follows. Consider a diagram $G$ involving only real frequencies. Assume, for definiteness, that the legs 1 and 2 are fakeons ${ }^{9}$ and complete the strategy outlined in subsection 2.5. Steps 1-4) give the spectral optical identities of the Feynman version of $G$ (where all the internal legs are prescribed à la Feynman). After that, we apply the fakeon prescription/projection of step 5).

Once these operations are concluded, we obtain the identities we start from to make the complexification. We write them as

$$
f(\omega)+\bar{f}(\omega)+\sum_{c} f_{c}(\omega)=0
$$

where $\omega$ is the frequency we want to complexify and $f(\omega), \bar{f}(\omega)$ and $f_{c}(\omega)$ are the contributions of the diagram itself, its complex conjugate and the cut diagrams, respectively. Note that, after step 5), the thresholds do not involve fakeon frequencies any longer. This is crucial, because it would make no sense to complexify frequencies that appear inside delta functions.

We first consider the complexification $\omega \rightarrow \Omega$ of the frequency $\omega$ associated with leg 1 . Since (11.5) holds for arbitrary $\omega$ (and $\omega$ does not enter the $\delta$ functions), we can replace it everywhere by the complex $\Omega$. The principal values may sound redundant, at this stage, but we keep them anyway, for reasons that become clear below. Let us also multiply by a complex factor $K$. This gives the relation

$$
K f(\Omega)+K \bar{f}(\Omega)+\sum_{c} K f_{c}(\Omega)=0
$$

with one caveat: the complex conjugation in $\bar{f}(\Omega)$ acts on the function $f$, but not its variable $\Omega$. This means that (11.6) cannot be interpreted as the spectral optical identity of the diagram with complexified frequencies, since $K \bar{f}(\Omega)$ is not the complex conjugate of $K f(\Omega)$. Indeed, a propagator with complex frequencies cannot come from a Hermitian theory, so it cannot lead to an optical theorem.

A second identity can be obtained from (11.5) by replacing $\omega$ everywhere with $\bar{\Omega}$ and multiplying by $\bar{K}$ :

$$
\bar{K} f(\bar{\Omega})+\bar{K} \bar{f}(\bar{\Omega})+\sum_{c} \bar{K} f_{c}(\bar{\Omega})=0 .
$$

\footnotetext{
${ }^{8}$ In this paper, these two operations are condensed into a unique operation, which is step 5) of subsection 2.5.

${ }^{9}$ We need two fakeons to highlight properties that are not visible with just one. The generalization from two to an arbitrary number is then straightforward.
} 
Summing (11.6) and (11.7), we get

$$
[K f(\Omega)+\bar{K} f(\bar{\Omega})]+[\bar{K} \bar{f}(\bar{\Omega})+K \bar{f}(\Omega)]+\sum_{c}\left[K f_{c}(\Omega)+\bar{K} f_{c}(\bar{\Omega})\right]=0,
$$

which is the desired identity. We want to show that the first bracket is the diagram with the propagator

$$
\frac{2 i K \Omega}{e^{2}-\Omega^{2}}+\frac{2 i \bar{K} \bar{\Omega}}{e^{2}-\bar{\Omega}^{2}}
$$

in leg 1 , the second bracket is the complex conjugate diagram and the sum collects the contributions of the cut diagrams. We go through the steps 1-5) of subsection 2.5 once again and generalize them to the case at hand. When we do so, we understand that leg 2 has been treated in a similar way. We distinguish the frequencies of the two fakeon legs by means of the subscripts 1 and 2 .

Let us start from step 2). Recalling how the residue theorem is applied to get to (11.5), we recognize that the integral on the loop energies with the propagator (11.9) must be performed along the LW integration path (see [15] or [16] for details), which is, by definition, the path that picks the residues we need. ${ }^{10}$

Moving to step 3), we need identities like (2.21) to eliminate the denominators $\tilde{D}_{\text {pseudo }}$. Those manipulations are legitimate only if the denominators never vanish, which is not guaranteed by a nonvanishing $M$. Indeed, we may find fractions like ${ }^{11}$

$$
\frac{1}{E-\Omega_{1}-\bar{\Omega}_{2}+i \epsilon},
$$

where $E$ is a linear combination of energies and possibly (real) frequencies. The denominator of (11.10) is singular in extended regions that may intersect the Minkowskian region (which is the real subspace $P_{R}$ of the space $P_{\text {ext }}$ of the complexified external momenta), as can be seen in the bubble diagram studied in ref. [16] (see also [8] and [13]). In particular, this may happen when the legs 1 and 2 propagate the same particle (same $\mu$ and same $M$ ). The $i \epsilon$ does not help and can be ignored here.

The point is that, as shown in [16], the LW integration path is an incomplete prescription. We need to complete it by deforming the integration domain on the space components of the loop momenta. The domain deformation generates Cauchy principal values inside the integrals $[8,16]$. Since the spectral optical identities concern the integrands, they formally do not change.

The algebraic manipulations that remove $\tilde{D}_{\text {pseudo }}$ are legitimate in the Euclidean region of $P_{\text {ext }}$, which extends to the Minkowskian subregion below each threshold: there the denominators of the integrands never vanish. The other regions are reached from the Euclidean one either analytically (if we need to cross a physical threshold or a fakeon

\footnotetext{
${ }^{10}$ Thick fakeons and LW decaying ghosts have the LW integration path in common, although they differ in the rest. The reason is that, as far as the integrals on the loop energies are concerned, the Feynman integration path, the integration path following from the Wick rotation and the LW integration path are all the same thing.

${ }^{11}$ This is the part of the argument where we need at least 2 fakeons.
} 
threshold that does not fall on $P_{R}$ ) or by means of the domain deformation (if we need to cross a fakeon threshold that falls on $P_{R}$ ).

Recapitulating, step 3) of subsection 2.5 is performed in the Euclidean region. As far as step 4) is concerned, we move from the Minkowskian subregion that lies below each threshold to the regions above the thresholds, reaching them one by one, in all directions.

When the threshold involves fakeon frequencies and falls on $P_{R}$, we proceed by means of the domain deformation, which incorporates also the fakeon projection of step 5). When the threshold is physical (i.e., it involves no fakeon frequencies), we apply the threshold decomposition as before. Finally, when the threshold involves fakeon frequencies and does not fall on $P_{R}$, it is not a true threshold (precisely because it is located away from $P_{R}$ ), so it poses no obstacle.

Next, we apply the manipulations of point 4.b) and finally step 5). Formally, the manipulations of step 4.b) remain the same, since the domain deformation, as recalled above, generates principal values inside the integrals on the space components of the loop momenta. To leave the previous formulas the same, it is convenient to extend the meaning of the principal value sign $\mathcal{P}$ to include the operations just described.

In the end, we get exactly (11.8). The procedure just outlined returns the correct diagram, as well as its complex conjugate and the cut diagrams. So doing, we have spectral optical identities for theories of particles and fakeons of all types.

The coincidence splitting method is also required in the presence of thick fakeons, to deal with powers of fractions like (11.10): since the domain deformation may generate principal values, powers of those may appear in involved diagrams.

If some situations it may be necessary to take into account the effects of the experimental apparatus on fakeons, like the energy resolution $\Delta E$ of the processes mediated by them. We can achieve this goal by turning an ordinary fakeon into a thick one, switching to a propagator of type (11.4) with $M=\Delta E$. Similarly, we can turn a thick fakeon into a thicker one by increasing $M$. Since $\Delta E$ does not enter the Lagrangian, it can have a different impact on different thresholds, which means that we can have a different $\Delta E$ in each level of the threshold decomposition of a diagram and each spectral optical identity (as long it is the same throughout the identity). Assuming an "optimized" experimental situation, we can take a nonzero $\Delta E$ only in the thresholds that concern the experiment we are making. For example, if we are studying the production of a $\mu^{+} \mu^{-}$pair and the theory includes a fakeon channel $\mathrm{f}_{\mathrm{c}}$ mediating that production, we just need $\Delta E$ in the contributions interested by $\mathrm{f}_{\mathrm{c}}$. Anywhere else, we can keep $\Delta E=0$ and use the coincidence splitting method, when needed. The spectral optical theorem continues to hold at $\Delta E \neq 0$.

Note that it is nontrivial to be able to include the energy resolution without violating the optical theorem. The interaction with the experimental apparatus is not required to be unitary, since the system can no longer be viewed as an isolated one.

\section{Massless fields and infrared divergences}

In this section we explain how to handle the threshold decomposition and the fakeon prescription in the presence of massless fields. 
Massless fields are responsible for infrared divergences in scattering amplitudes [1821]. The divergences only appear on shell, when we integrate on the space components of the loop momenta and the phase spaces. To identify potential sources of divergences, we study the infrared behaviors of the skeletons. We first discuss the issue with physical particles only, then include fakeons. For definiteness, we assume that the massless fields are photons. Collinear gluons and gravitons can be treated the same way (with angular resolutions and other types of resolutions, in addition to the energy resolutions).

The diagram $G$ and its conjugate $\bar{G}$ are well defined, since they are off-shell, but the cut diagrams $G_{c}$ may not be separately well defined, since the cuts put various particles on shell. The cancellation of the infrared divergences in the sum

$$
\sum_{c} G_{c}=-G-\bar{G}
$$

is due to the usual compensation between soft and virtual photons. We can regulate each $G_{c}$ by equipping the photons with small fictitious masses $m$. Then we obtain the identity

$$
\sum_{c} G_{c}(m)=-G(m)-\bar{G}(m)
$$

with self-evident notation.

The energy resolution $\Delta E_{\mathrm{IR}}$ of the detectors can be taken into account to make the limits $m \rightarrow 0$ well defined. If a cut diagram $G_{c}(m)$ involves the integral on the phase space of a given photon $\gamma$, with momentum $\mathbf{k}_{\gamma}$ and mass $m_{\gamma}$, we write it as $G_{c}^{<}(m)+G_{c}^{>}(m)$, where $G_{c}^{<}(m)$ is the integral on $\left|\mathbf{k}_{\gamma}\right| \leqslant \Delta E_{\mathrm{IR}}$ and $G_{c}^{>}(m)$ is the integral on $\left|\mathbf{k}_{\gamma}\right| \geqslant \Delta E_{\mathrm{IR}}$. If the leg $\gamma$ is uncut in $G_{c}(m)$, we take $G_{c}^{<}(m)=G_{c}(m)$ and $G_{c}^{>}(m)=0$. By construction, $G_{c}^{>}(m)$ has a well-defined limit $m_{\gamma} \rightarrow 0$. We obtain

$$
G(m)+\bar{G}(m)+\sum_{c} G_{c}^{>}(m)+\sum_{c} G_{c}^{<}(m)=0 .
$$

In the presence of massless fields, we cannot imagine the asymptotic state of a particle (such as the electron) as just made of the particle itself. We have to think of it as made of the particle and a cloud of soft photons radiated by it. Guided by this, we can attach $G_{c}^{<}(m)$ to some other $G_{c^{\prime}}^{<}(m)$ and reorganize the last sum of (12.3) into a sum of terms with well-defined limits $m_{\gamma} \rightarrow 0$. We can also view the last sum as a unique, convergent contribution. We repeat this construction for all the photons. At the end, the limit where the photons become massless gives the optical theorem in the presence of massless fields.

Now, consider the case of massless fields $\varphi_{0}$ in the presence of fakeons. The fields $\varphi_{0}$ themselves must be physical (not fakeons). Indeed, massless fakeons are excluded, because they violate causality at arbitrarily large distances (instead of just microcausality, see for example [9]). As before, we equip each $\varphi_{0}$ with a small fictitious mass $m$. In the massless limit $m \rightarrow 0$, some (multi)thresholds $\Delta_{i}$ that are distinct at $m \neq 0$ may tend to the same threshold $\Delta_{0}$ for low $\varphi_{0}$ energies. ${ }^{12}$ This means that the $\Delta_{i}$ can interfere with one another

\footnotetext{
${ }^{12}$ For example, consider the second diagram of figure 5 with $\varphi_{0}$ in the vertical internal line and identical massive fields in the other internal lines. We can view this case as the third diagram of figure 4 with $m_{1}=m_{2}=m_{4}=m_{5}, m_{3}=0$ and $p_{3}=p_{1}+p_{4}=p_{2}+p_{5}$. Then it easy to check that $\Delta^{135} \simeq \Delta^{12}$ for low $\varphi_{0}$ energies $\left(\omega_{3} \simeq 0\right)$.
} 
after the massless limit, even if they do not interfere before. In section 10 we have shown that the limit of coinciding thresholds is regular, because it allows us to define powers of principal values and $\delta$ functions. ${ }^{13}$

We know that the starting uncut diagram $G$ (defined by the Feynman prescription everywhere) does not have infrared divergences. This is enough to guarantee that the every threshold $\Delta_{0}$ of its threshold decomposition is separately convergent. The reason is that the potentially divergent behaviors of different levels cannot compensate one another, for example through identities such as (2.19). Indeed, the infrared divergences, like the ultraviolet ones, are due to power counting behaviors of the integrands and do not have imaginary parts. Without imaginary parts, they cannot propagate to lower or higher levels.

Thus, the compensations must occur among the contributions of the same level. Ultimately, they occur within the same threshold $\Delta_{0}$, because each threshold is independent.

What happens can be described as follows. We know that $\Delta_{0}$ can come from different thresholds $\Delta_{i}$ of the $m \neq 0$ diagram $G(m)$. Each $\Delta_{i}$ can separately have infrared divergences in the limit $m \rightarrow 0$. However, $G$ is free of them, so the infrared divergences due to $\Delta_{i}$ must cancel out in $\Delta_{0}$.

Moreover, if $\Delta_{0}$ contains a fakeon frequency, all the thresholds $\Delta_{i}$ it comes from contain fakeon frequencies: since the massless field $\varphi_{0}$ is not a fakeon, it cannot change the nature (physical vs fake) of the thresholds it enters. If two thresholds $\Delta_{i}$ tend to coincide for low $\varphi_{0}$ energies in the $m \rightarrow 0$ limit, they must either be both physical or both fake. Therefore, the cancellation of infrared divergences survives the fakeon prescription/projection and the limit $m \rightarrow 0$, as we wished to show. The optical theorem, in the form explained above, continues to hold.

The cancellation need not occur if the massless field is a fakeon. The argument just given cannot guarantee that all the $\Delta_{i}$ are treated the same by the fakeon projection in that case.

\section{Conclusions}

We have shown that the whole unitarity problem in quantum field theory can be reduced to a set of algebraic identities, which do not require to integrate on the space components of the loop momenta, or the phase spaces in cut diagrams, and hold for each threshold separately, for diagrams with arbitrary derivative vertices (as long as the Lagrangian of the theory is Hermitian) and for propagators with arbitrary residues, masses and frequencies.

The key ingredient is a proper threshold decomposition, since different thresholds do not interfere with one another. First, we integrate on the loop energies by means of the residue theorem, which is an algebraic operation. Second, we ignore the integrals on the space components of the loop momenta or phase spaces and work on the skeleton of the diagram. Third, we eliminate the pseudothresholds, which are unphysical, because they involve differences of frequencies. Fourth, we perform the threshold decomposition, which

\footnotetext{
${ }^{13}$ There, we had powers of principal values and $\delta$ functions with the same momenta. In the case of coinciding thresholds due to the massless limit $m \rightarrow 0$, the powers originate from different loops. The extra loop integrals have further smearing effects.
} 
provides a separate optical identity for each (multi)threshold. Fifth, we drop the thresholds involving frequencies associated with the legs that we want to quantize as fakeons.

The threshold decomposition must be done with due care. For example, it is not known a priori how to extract the purely virtual content of a diagram, which is defined by the procedure itself.

The gain in insight is important. The spectral optical identities hold for thick as well as non thick fakeons, with arbitrary residues on the poles of the propagators. We can treat massless physical fields and even introduce certain details of the experimental apparatus, like the energy resolution around the fakeon peak, without violating the identities and, ultimately, unitarity. In the whole analysis we never need to make a single non algebraic operation.

Summing the spectral optical identities associated with a loop diagram, we derive the spectral optical theorem obeyed by its skeleton. As soon as we resume the integrals on the space components of the loop momenta and the phase spaces, we obtain the usual optical theorem for amplitudes.

We have given explicit examples to show how the threshold decomposition works and how the spectral optical identities are derived. At one loop we have analyzed the bubble, the triangle, the box, the pentagon and the hexagon. At two loops we have studied the triangle with "diagonal" and the box with diagonal. Whole classes of diagrams with arbitrarily many loops are included straightforwardly. The calculations provide formulas for the loop integrals with fakeons and relate them to the known formulas for the loop integrals with physical particles. It is possible to use the results of this paper to implement the fakeon prescription in softwares like FeynCalc, FormCalc, LoopTools and Package-X.

Finally, the strategy we have elaborated also provides a general proof of the identities for arbitrary diagrams.

\section{Acknowledgments}

We are grateful to U. Aglietti, L. Marzola, M. Piva and M. Raidal for helpful discussions and the Laboratory of High Energy and Computational Physics of NICPB (National Institute of Chemical Physics and Biophysics), Tallinn, Estonia, for hospitality during the first part of this work.

Open Access. This article is distributed under the terms of the Creative Commons Attribution License (CC-BY 4.0), which permits any use, distribution and reproduction in any medium, provided the original author(s) and source are credited.

\section{References}

[1] R.E. Cutkosky, Singularities and discontinuities of Feynman amplitudes, J. Math. Phys. 1 (1960) 429 [inSPIRE].

[2] M.J.G. Veltman, Unitarity and causality in a renormalizable field theory with unstable particles, Physica 29 (1963) 186 [INSPIRE]. 
[3] G. 't Hooft, Renormalization of massless Yang-Mills fields, Nucl. Phys. B 33 (1971) 173 [INSPIRE].

[4] G. 't Hooft, Renormalizable Lagrangians for massive Yang-Mills fields, Nucl. Phys. B 35 (1971) 167 [InSPIRE].

[5] G. 't Hooft and M.J.G. Veltman, Diagrammar, CERN-73-09, CERN, Geneva, Switzerland (1974) [NATO Sci. Ser. B 4 (1974) 177] [InSPIRE].

[6] M. Veltman, Diagrammatica. The path to Feynman rules, Cambridge University Press, New York, NY, U.S.A. (1994).

[7] D. Anselmi, On the quantum field theory of the gravitational interactions, JHEP 06 (2017) 086 [arXiv:1704.07728] [INSPIRE].

[8] D. Anselmi, Fakeons and Lee-Wick models, JHEP 02 (2018) 141 [arXiv:1801.00915] [INSPIRE].

[9] D. Anselmi, Fakeons, microcausality and the classical limit of quantum gravity, Class. Quant. Grav. 36 (2019) 065010 [arXiv: 1809.05037] [INSPIRE].

[10] T.D. Lee and G.C. Wick, Negative metric and the unitarity of the $S$ matrix, Nucl. Phys. B 9 (1969) 209 [inSPIRE].

[11] T.D. Lee and G.C. Wick, Finite theory of quantum electrodynamics, Phys. Rev. D 2 (1970) 1033 [INSPIRE].

[12] T.D. Lee, A relativistic complex pole model with indefinite metric, in Quanta: essays in theoretical physics dedicated to Gregor Wentzel, Chicago University Press, Chicago, IL, U.S.A. (1970), pg. 260.

[13] N. Nakanishi, Lorentz noninvariance of the complex-ghost relativistic field theory, Phys. Rev. D 3 (1971) 811 [INSPIRE].

[14] R.E. Cutkosky, P.V. Landshoff, D.I. Olive and J.C. Polkinghorne, A non-analytic S matrix, Nucl. Phys. B 12 (1969) 281 [INSPIRE].

[15] B. Grinstein, D. O'Connell and M.B. Wise, Causality as an emergent macroscopic phenomenon: the Lee-Wick $O(N)$ model, Phys. Rev. D 79 (2009) 105019 [arXiv:0805.2156] [INSPIRE].

[16] D. Anselmi and M. Piva, A new formulation of Lee-Wick quantum field theory, JHEP 06 (2017) 066 [arXiv:1703.04584] [INSPIRE].

[17] D. Anselmi and M. Piva, Perturbative unitarity of Lee-Wick quantum field theory, Phys. Rev. D 96 (2017) 045009 [arXiv:1703.05563] [inSPIRE].

[18] F. Bloch and A. Nordsieck, Note on the radiation field of the electron, Phys. Rev. 52 (1937) 54 [INSPIRE].

[19] T. Kinoshita, Mass singularities of Feynman amplitudes, J. Math. Phys. 3 (1962) 650 [INSPIRE].

[20] T.D. Lee and M. Nauenberg, Degenerate systems and mass singularities, Phys. Rev. 133 (1964) B1549 [INSPIRE].

[21] S. Weinberg, Infrared photons and gravitons, Phys. Rev. 140 (1965) B516 [InSPIRE].

[22] D. Anselmi, The quest for purely virtual quanta: fakeons versus Feynman-Wheeler particles, JHEP 03 (2020) 142 [arXiv:2001.01942] [INSPIRE]. 
[23] D. Anselmi, E. Bianchi and M. Piva, Predictions of quantum gravity in inflationary cosmology: effects of the Weyl-squared term, JHEP 07 (2020) 211 [arXiv:2005.10293] [INSPIRE].

[24] D. Anselmi et al., Phenomenology of a fake inert doublet model, JHEP 10 (2021) 132 [arXiv: 2104.02071] [INSPIRE].

[25] D. Anselmi et al., Fake doublet solution to the muon anomalous magnetic moment, Phys. Rev. D 104 (2021) 035009 [arXiv:2104.03249] [InSPIRE].

[26] G.J. van Oldenborgh and J.A.M. Vermaseren, New algorithms for one loop integrals, Z. Phys. C 46 (1990) 425 [inSPIRE].

[27] J. Kublbeck, M. Böhm and A. Denner, Feyn Arts: computer algebraic generation of Feynman graphs and amplitudes, Comput. Phys. Commun. 60 (1990) 165 [INSPIRE].

[28] A. Denner, Techniques for calculation of electroweak radiative corrections at the one loop level and results for W physics at LEP-200, Fortsch. Phys. 41 (1993) 307 [arXiv:0709.1075] [INSPIRE].

[29] T. Hahn, Loop calculations with FeynArts, FormCalc, and LoopTools, Acta Phys. Polon. B 30 (1999) 3469 [hep-ph/9910227] [INSPIRE].

[30] T. Hahn, Generating Feynman diagrams and amplitudes with FeynArts 3, Comput. Phys. Commun. 140 (2001) 418 [hep-ph/0012260] [INSPIRE].

[31] A. Alloul, N.D. Christensen, C. Degrande, C. Duhr and B. Fuks, FeynRules $2.0-a$ complete toolbox for tree-level phenomenology, Comput. Phys. Commun. 185 (2014) 2250 [arXiv: 1310.1921] [INSPIRE].

[32] H.H. Patel, Package-X: a Mathematica package for the analytic calculation of one-loop integrals, Comput. Phys. Commun. 197 (2015) 276 [arXiv:1503.01469] [INSPIRE].

[33] D. Anselmi, Algebraic cutting equations, Annals Phys. 394 (2018) 294 [arXiv:1612.07148] [INSPIRE].

[34] R.E. Cutkosky, Anomalous thresholds, Rev. Mod. Phys. 33 (1961) 448.

[35] R.J. Eden, P.V. Landshoff, D.I. Olive and J.C. Polkinghorne, The analytic S matrix, Cambridge University Press, Cambridge, U.K. (1966).

[36] G. Passarino and M.J.G. Veltman, One loop corrections for $e^{+} e^{-}$annihilation into $\mu^{+} \mu^{-}$in the Weinberg model, Nucl. Phys. B 160 (1979) 151 [inSPIRE].

[37] C.G. Bollini and J.J. Giambiagi, Lowest order "divergent" graphs in $\nu$-dimensional space, Phys. Lett. B 40 (1972) 566 [INSPIRE].

[38] G. 't Hooft and M.J.G. Veltman, Regularization and renormalization of gauge fields, Nucl. Phys. B 44 (1972) 189 [inSPIRE].

[39] G.M. Cicuta and E. Montaldi, Analytic renormalization via continuous space dimension, Lett. Nuovo Cim. 4 (1972) 329 [INSPIRE].

[40] D. Anselmi, Fakeons and the classicization of quantum gravity: the FLRW metric, JHEP 04 (2019) 061 [arXiv:1901.09273] [INSPIRE]. 